\title{
The lichens of Moshchny Island (Lavansaari) - one of the remote islands in the Gulf of Finland
}

\author{
Irina S. Stepanchikova ${ }^{1,2}$, Dmitry E. Himelbrant ${ }^{1,2}$, Ulf Schiefelbein ${ }^{3}$, Jurga \\ Motiejūnaité $^{4}$, Teuvo Ahti ${ }^{5}$, Mikhail P. Andreev ${ }^{2}$ \\ ${ }^{1}$ St. Petersburg State University, Universitetskaya emb. 7-9, 199034 St. Petersburg, Russia. \\ E-mails: stepa_ir@mail.ru,d_brant@mail.ru \\ ${ }^{2}$ Laboratory of Lichenology and Bryology, Komarov Botanical Institute RAS, Professor Popov St. 2, \\ 197376 St. Petersburg, Russia. E-mail: andreevmp@binran.ru \\ ${ }^{3}$ Blücherstrasse 71, D-18055 Rostock, Germany. E-mail: Ulf.Schiefelbein@gmx.de \\ ${ }^{4}$ Laboratory of Mycology, Institute of Botany, Nature Research Centre, Žaliųjų Ežerų 49, LT-08406 Vilnius, Lithuania. \\ E-mail: jurga.motiejunaite@gamtc.lt \\ ${ }^{5}$ Botanical Museum, Finnish Museum of Natural History, University of Helsinki, P.O. Box 7, FI-00014 Helsinki, Finland. \\ E-mail: teuvo.ahti@helsinki.fi
}

\begin{abstract}
We present a checklist for Moshchny Island (Leningrad Region, Russia). The documented lichen biota comprises 349 species, including 313 lichens, 30 lichenicolous fungi and 6 non-lichenized saprobic fungi. Endococcus exerrans and Lichenopeltella coppinsii are reported for the first time for Russia; Cercidospora stenotropae, Erythricium aurantiacum, Flavoplaca limonia, Lecidea haerjedalica, and Myriospora myochroa for European Russia; Flavoplaca oasis, Intralichen christiansenii, Nesolechia fusca, and Myriolecis zosterae for North-Western European Russia; and Arthrorhaphis aeruginosa, Calogaya pusilla, and Lecidea auriculata subsp. auriculata are new for Leningrad Region. The studied lichen biota is moderately rich and diverse, but a long history of human activity likely caused its transformation, especially the degradation of forest lichen biota. The most valuable habitats for lichens in Moshchny Island are seashore and dune communities which definitely deserve protection.
\end{abstract}

Keywords: Baltic Sea, Karelia australis, Leningrad Region, dune communities, Endococcus exerrans, Lichenopeltella coppinsii

\section{INTRODUCTION}

Moshchny Island (Lavansaari in Finnish) is one of largest remote islands in the Russian part of the Gulf of Finland within the boundaries of Leningrad Region. It was permanently inhabited for long periods since the $14^{\text {th }}$ century and had regular ship connection with the mainland since the $19^{\text {th }}$ century. However, it remained practically uninvestigated by lichenologists. The earliest known lichen collections from the island were made by Edvin Nylander in 1851 (two specimens, Hypogymnia physodes and Stereocaulon glareosum) and Magnus Brenner in July 1868 (a specimen of Amandinea punctata and two specimens of Ramalina subfarinacea), all now kept in the lichen collection in the Finnish Museum of Natural History, University of Helsinki (H). They were first identified by William Nylander and later critically revised by Nadezhda M. Alexeeva and Dmitry E. Himelbrant in 2007-2008. In 1926 Bertel Lemberg investigated plant communities on dunes and mentioned 4 species of terricolous lichens - Cladonia arbuscula, C. cornuta, C. rangiferina and Stereocaulon paschale from the island (Lemberg, 1935), and in 1993 Nataliya B. Balashova collected samples of 12 common corticolous species, which were subsequently identified by Alexeeva (LECB; Alexeeva, 2005). All the mentioned collections and reports contain very scarce and fragmentary information on lichens. Only 19 lichen species were known from Moshchny Island before our survey.

Irina Stepanchikova (IS) and Dmitry Himelbrant (DH) conducted a comprehensive field study of Moshchny lichens in 2017 in connection with the Complex Expedition 'Gogland' of the Russian Geographical Society. The participants of the same expedition Nadezhda S. Liksakova and Elena A. Glazkova, who investigated the vegetation and flora of Moshchny Island, recently mentioned the seven most common terricolous lichens (Liksakova \& Glazkova, 2018), identified by IS and DH. The present paper is the outcome of the expedition and the revision of all known herbarium collections and literature records from Lavansaari. 


\section{STUDY AREA}

Moshchny Island lies in the Russian part of the Gulf of Finland, c. $30 \mathrm{~km}$ NW of the Kurgal'sky Peninsula (Russia) and $70 \mathrm{~km} \mathrm{SW}$ of Kotka (Finland), and occupies an area of c. $16 \mathrm{~km}^{2}$ (including 7 very small in-shore islets). Administratively it belongs to the Kingisepp District of the Leningrad Region, and biogeographically to Karelia australis, a traditional province of the Eastern Fennoscandia (Kotiranta et al., 1998).
The island consists of the main part and the large Promezhutochny Peninsula (former Suisaari), connected by the 200-400 m wide and 1 $\mathrm{km}$ long, sandy isthmus Hiekkataipale (Fig. 1). The relief of the island is represented by rather low flatland (average height is less than $10 \mathrm{~m}$ a.s.1.) with two local maximal elevations of 16 and $12 \mathrm{~m}$ a.s.1. The whole island is composed of sedimentary (marine) and moraine sand with granite moraine boulders. Rocky outcrops are

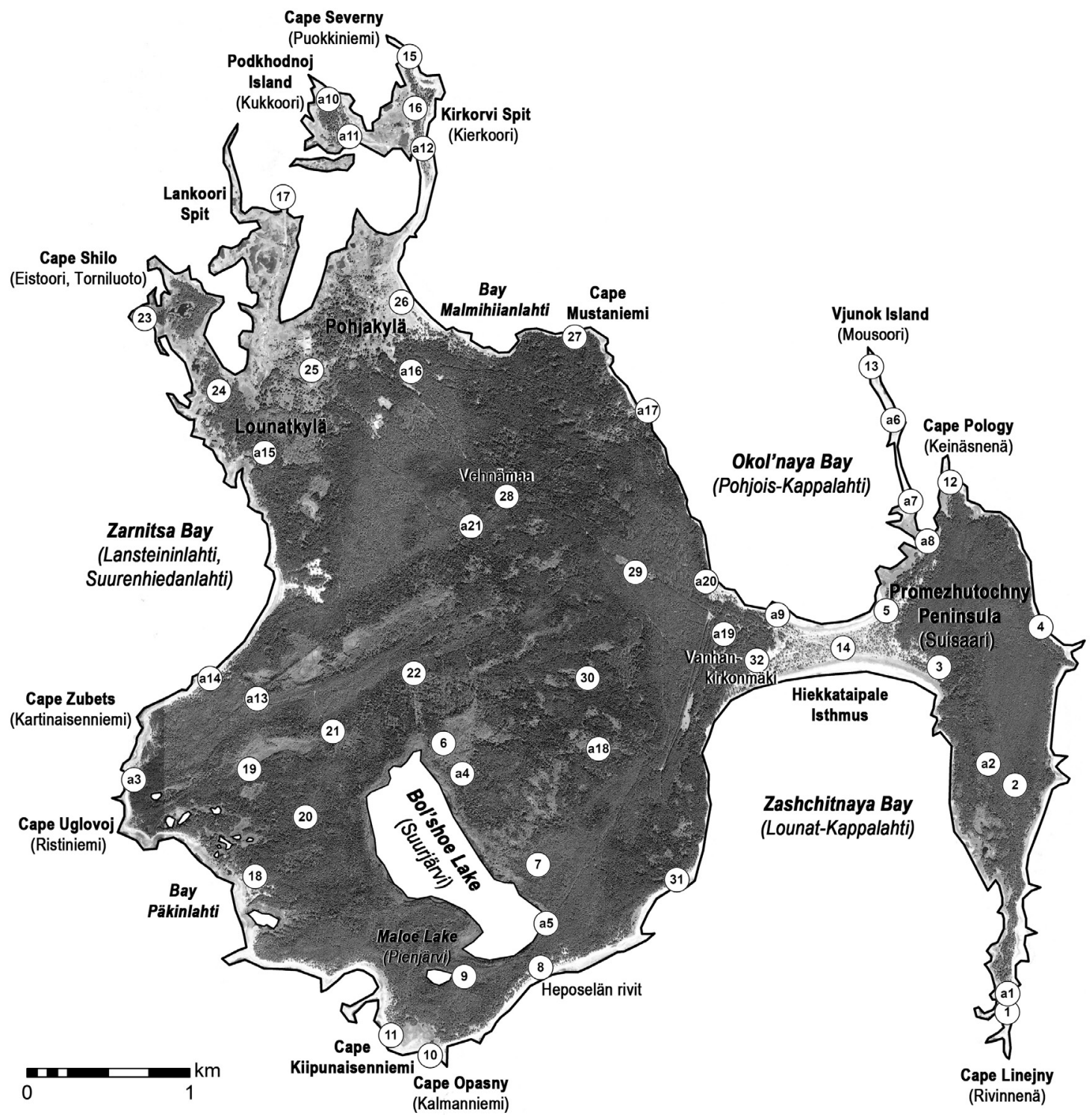

Fig. 1. The study area, Moshchny Island (Lavansaari), with location of collection sites. 
totally absent. A significant part of Moshchny Island is covered by pine forests on sand (mainly in $\mathrm{W}, \mathrm{S}$, and $\mathrm{N}$ parts of island); spruce, smallleaved, and black alder forests on clayey soil are also widely distributed (mainly in the central, E and $\mathrm{N}$ parts). Almost all forests nowadays are secondary and composed of young stands, as practically the whole territory of the island has undergone anthropogenic transformation. Dune communities (in E and $\mathrm{W}$ parts), heathlands, and different types of seashore communities are characteristic for the island (Liksakova \& Glazkova, 2018).

In the $14^{\text {th }}$ century the island was colonized by Swedish-speaking fishermen and seal hunters (its Swedish name is Lövskär). However, the island was deserted again in the 1570s due to a long war between Sweden and Russia. From 1630 s another permanent colonization started, this time from the Finnish coast in the north. In 1920-1940 the island belonged to the state of Finland (Piispa, 2013). Gradually a community of up to 1300 inhabitants was formed. In addition to fishing, ship-building and commercial shipping were the main means of living. In the late 19th century lots of sand and stones were sold to St. Petersburg, e. g., to the construction of the Kronstadt fortification. Maintenance of cattle and horses (important for wintertime fishing) meant that also some cultivated fields were present. There was also a fish-processing plant, and tourist industry was being developed in the 1930s. Connections to Estonia were also frequent. Before the World War I some military fortification occurred, as again during and after the World War II, even with an airfield and narrow gauge railway. The civil population was evacuated to Finland in 1939, and after that the island has had very few permanent inhabitants (Piispa, 2013; Liksakova \& Glazkova, 2018).

\section{MATERIAL AND METHODS}

The material was collected on Moshchny Island from 12 to 22 of August, 2017 by IS and $\mathrm{DH}$. Altogether 53 localities were investigated (Appendix 1; Fig. 1): 32 standard $20 \times 20 \mathrm{~m}$ sample areas (or in natural boundaries of the community), where the lichen diversity on each substrate was described as detailed as possible, and 21 additional plots, where only individual units of substrates and species were recorded.
The specimens are deposited in the herbaria of the Botanical Museum, University of Helsinki $(\mathrm{H})$, the Department of Botany, St. Petersburg State University (LECB), and the Institute of Botany, Nature Research Centre in Vilnius (BILAS). The list of localities also includes two localities mentioned by Lemberg in his publication. Other herbarium and literature records are indicated as 'Lavansaari' or 'Moshchny Island' without further details. IS and DH identified the majority of the specimens, Jurga Motiejūnaite performed identification of several lichenicolous fungi, Ulf Schiefelbein identified some lichenicolous fungi and seashore saxicolous lichens, Teuvo Ahti identified or confirmed critical Cladonia specimens, Mikhail Andreev identified Lecidea haerjedalica and some other crustose saxicolous lichens; if otherwise, the author responsible for the identification is indicated.

In the present list of species the nomenclature of taxa generally follows Nordin et al. (2011), Hafellner \& Türk (2016), Diederich et al. (2018), and Lawrey \& Diederich (2018). For each species the substrates and localities are mentioned. Species new to the Leningrad Region or larger regions are accompanied by information on diagnostic characteristics and distribution in North-Western European Russia, Fennoscandia and the Baltic countries. Lichen substances are given for HPTLC-analyzed species. Chromatography was performed by IS \& DH (different taxa), as well as Ludmila Konoreva \& Sergei Chesnokov (Micarea spp.) according to standard techniques of high performance thin-layer chromatography using solvent systems A, B, and C (Orange et al., 2001). Lichenicolous and algicolous fungi are marked with \#, non-lichenized fungi with +, habitat specialists with ${ }^{\text {s }}$, and indicator species with ${ }^{\mathbf{i}}$ (see Andersson et al., 2009); regions are abbreviated as follows: LR - Leningrad Region, ELR - Eastern Leningrad Region, WLR - Western Leningrad Region, SPb - St. Petersburg; collectors are abbreviated as follows: MB - Magnus Brenner, NB - Nataliya B. Balashova, EN - Edvin Nylander. For each species collected by the authors, the frequency of occurrence is indicated in square brackets: single record $\left[R_{1}\right]$ - the species was found once, rare $[R]$ - recorded in 2-6 standard sample areas or more than in one additional plot, occasionally [O] $-7-12$, frequent $[\mathrm{F}]-13-19$, common $[\mathrm{C}]-20-25$, very common [VC] - 26-32 standard sample areas. 


\section{THE SPECIES}

ABsconditella lignicola Vězda \& Pišút - on wood of Pinus sylvestris L.; $27\left[\mathrm{R}_{1}\right]$.

ACAROSPORA FUSCATA (Schrad.) Th. Fr. - on granite boulders; 1, 7, 12, 15, 16, 18, 23, 25, 26, a16 [O].

ACAROSPORA GLAUCOCARPA (Ach.) Körb. - on concrete; a10 $\left[\mathrm{R}_{1}\right]$.

ACAROSPORA MOENIUM (Vain.) Räsänen - on concrete, slate; a10, a19, a21 [R].

ACAROSPORA VERonensis A. Massal. - on granite boulders and iron; 8, a1 [R].

s ACROCORDIA CAVATA (Ach.) R. C. Harris - on bark of Populus tremula L.; 22, a20 [R]. Red Data Book of LR (Red..., 2018).

Amandinea CACUminum (Th. Fr.) H. Mayrhofer \& Sheard - on wood, granite boulders, iron; 13, 15, 16 [R].

Amandinea Coniops (Wahlenb.) M. Choisy ex Scheid. \& H. Mayrhofer - on granite boulders; $1\left[\mathrm{R}_{1}\right]$.

AMANDinea PUnCTATA (Hoffm.) Coppins \& Scheid. - on bark of Acer platanoides L., Juniperus communis L., Sorbus aucuparia L., wood, granite boulders, concrete, bones; 1, 10, 13, 15, 16, 25, 27, 31, a3, a9, a10 [O]. Coll. MB: on bark of deciduous tree, 27.07.1868, det. W. Nylander as Lecidea myriocarpa (DC.) (H s. n.).

ANAPTYCHIA CILIARIS (L.) Körb. - on bark of Populus tremula; $22\left[\mathrm{R}_{1}\right]$.

ANISOMERIDIUM POLYPORI (Ellis \& Everh.) M. E. Barr - on bark of Acer platanoides; 25 [R $]$.

\# ARTHONIA EPIPHYSCIA Nyl. - on thallus of Physcia dubia on concrete manured by birds; $17\left[\mathrm{R}_{1}\right]$.

ARTHONIA MEDIELLA Nyl. - on bark of Quercus robur L.; a15 $\left[\mathrm{R}_{1}\right]$.

ARTHONia PATEllulata Nyl. - on bark of Populus tremula; a20 $\left[\mathrm{R}_{1}\right]$.

+ ARTHONIA PUNCTIFORMis Ach. - on bark of Alnus glutinosa (L.) Gaertn., Sorbus aucuparia; 6, 27, a7 [R].

ARTHONIA RADIATA (Pers.) Ach. - on bark of Sorbus aucuparia; 6, 27 [R].

i ARTHOnia SPADicEA Leight. - on bark of Alnus glutinosa; 6, 22, 30 [R]. Red Data Book of LR (Red..., 2018).

\# Arthrorhaphis aeruginosa R. Sant. \& Tønsberg - on thallus of Cladonia cf. cervicornis on soil; $8\left[\mathrm{R}_{1}\right](\mathrm{H})$. - New to LR. Distribution in North-Western European Russia outside of LR: Republic of Karelia (Fadeeva et al.,
2007). Distribution in Fennoscandia and Baltic countries: Norway, Sweden (Nordin et al., 2011), Estonia (Randlane et al., 2018), Lithuania (Motiejūnaitè, 2017). A lichenicolous ascomycete causing typical aeruginose colour of the basal squamules and suppression of podetia development of Cladonia species (Santesson \& Tønsberg, 1994).

ASPICILIA CINEREA (L.) Körb. - on granite boulders; $1,12,15,25,26,31$, a 16 [R].

Athallia Holocarpa (Hoffm.) Arup, Frödén \& Søchting - on concrete, slate, bones; 26, a3, a10, a12, a19, a21 [R].

AtHAllia PYRACEA (Ach.) Arup, Frödén \& Søchting - on bark of Alnus glutinosa, Juniperus communis, Populus tremula; 16, 22, a7, a20 [R].

AtHAllia scopUlaris (Nyl.) Arup, Frödén \& Søchting - on granite boulders and concrete; 1 , $10,13,15,17,31$, a10 [R].

\# ATHELIA ARACHNOIDEA (Berk.) Jülich - on crustose lichens and algae on bark of trees and concrete; a21 [ $\left.\mathrm{R}_{1}\right]$.

BACIDIA BAGLIETTOANA (A. Massal. \& De Not.) Jatta - on mosses on concrete; $26\left[\mathrm{R}_{1}\right]$.

BACIDINA PHACODES (Körb.) Vězda - on bones; a $14\left[R_{1}\right]$.

BAEOMYCES CARNEUS Flörke - on primary soil on granite boulder; 7 , a17 [R].

BAEOMYCES RUfus (Huds.) Rebent. - on brick; $29\left[R_{1}\right]$.

Bellemerea cinereorufescens (Ach.) Clauzade \& Cl. Roux - on granite boulders; $29\left[\mathrm{R}_{1}\right]$.

Biatora Albohyalina (Nyl.) Bagl. \& Carestia - on bark of Quercus robur, a15 [ $\left.\mathrm{R}_{1}\right]$.

BiATORA EFFLORESCENS (Hedl.) Räsänen - on bark of Alnus glutinosa, Picea abies (L.) H. Karst., Populus tremula, Quercus robur, Sorbus aucuparia; 6, 9, 22, 27, 30, a15 [R].

Biatora GLOBUlosa (Flörke) Fr. - on bark of Quercus robur; a15 $\left[\mathrm{R}_{1}\right]$.

Biatora Helvola Körb. ex Hellb. - on bark of Alnus glutinosa; $6\left[\mathrm{R}_{1}\right]$.

BiATORA OCELliformis (Nyl.) Arnold - on bark of Sorbus aucuparia; $27\left[\mathrm{R}_{1}\right]$.

Bilimbia sabuletorum (Schreb.) Arnold - on mosses on granite and concrete; 25, a16 [R].

BRIANARIA SYLVICOLA (Flot. ex Körb.) S. Ekman \& M. Svensson - on granite boulders, bricks, iron; 7, 29, a2, a16 [R].

BRYORIA CAPILlARIS (Ach.) Brodo \& D. Hawksw. - on bark of Alnus glutinosa, Picea abies, Pinus sylvestris, wood of Picea abies; 2, 20, 21, 28, 30 [R]. 
BRYoRIA FUsCEscens (Gyeln.) Brodo \& D. Hawksw. - on bark of Alnus glutinosa, Juniperus communis, Picea abies, Pinus sylvestris, wood of Picea abies and Pinus sylvestris, granite boulders; 16, 19-21, 23, 24, 27, 28, 30 [O].

BUELLIA GRISEOVIRENS (Turner \& Borrer ex Sm.) Almb. - on bark of Alnus glutinosa, Betula pubescens Ehrh., Quercus robur, Sorbus aucuparia, wood; 6, 9, 13, 15, 22, 26, 28, a9, a15 [O]. Thalli contain atranorin, norstictic acid, and traces of connorstictic acid.

BUELlia SCHAERERI De Not. - on bark of Picea abies, wood of Pinus sylvestris; 27 [ $\left.\mathrm{R}_{1}\right]$.

Calicium glaucellum Ach. - on wood of Pinus sylvestris; 24, a20 [R].

${ }^{i}$ Calicium tigillare (Ach.) Pers. - on wood; 16 [R $\left.{ }_{1}\right]$. Red Data Book of LR (Red..., 2018).

Calicium trabinellum (Ach.) Ach. - on wood of Picea abies; $19\left[\mathrm{R}_{1}\right]$.

Calicium viride Pers. - on bark of Picea abies; $27,30[R]$.

Calogaya pusilla (A. Massal.) Arup, Frödén \& Søchting - on concrete; 17 , a $10[\mathrm{R}](\mathrm{H}$, LECB). - New to LR, previously known from SPb (Himelbrant et al., 2015). This is a member of the Caloplaca saxicola group. It is very common on mortar and concrete and easily distinguished from other species by its almost always-present white pruina (Gaya, 2009). In the past this species was probably often published under the name C. saxicola. Here it is reported also from neighboring Bol'shoy Tuters Island (former Tytärsaari): SW shore, S of ponds Römenlammet, $59^{\circ} 50$ '09' $\mathrm{N}, 27^{\circ} 11^{\prime} 18^{\prime \prime} \mathrm{E}$, boulders of old quay, 28.05.2015, leg. IS (H); SW shore, $\mathrm{N}$ of ponds Römenlammet, $59^{\circ} 50^{\prime} 13$ ” N, $27^{\circ} 11^{\prime} 17^{\prime \prime} \mathrm{E}$, young aspen stand, on old concrete basement, 28.05.2015, leg. IS (H).

CALOPlaca ChlORINa (Flot.) H. Olivier - on concrete; $26\left[R_{1}\right]$.

CALOPLACA SAXICOLA (Hoffm.) Nordin - on concrete; $17\left[R_{1}\right]$.

Candelariella aurella (Hoffm.) Zahlbr. - on wood, granite boulders, concrete, slate, bones; 17, 26, a3, a10, a14, a17, a19 [R].

CAndelariella coralliza (Nyl.) H. Magn. - on wood and granite boulders; 15, 16, 26, 31 $[R]$.

Candelariella efflorescens R. C. Harris \& W. R. Buck - on bark of Acer platanoides; 25 [ $\left.R_{1}\right]$.

CANDElariella lutella (Vain.) Räsänen - on bark of Populus tremula; $9\left[\mathrm{R}_{1}\right]$.
Candelariella vitellina (Hoffm.) Müll. Arg. - on granite boulders, iron; $11,12,16,23,25$, 26, 31, a1 [O].

CAndelariella Xanthostigma (Ach.) Lettau - on bark of Acer platanoides, Populus tremula; $9,25[R]$.

CAtillaria chalybeia (Borrer) A. Massal. - on granite boulders; 1, 12, 26, 31 [R].

CATILlaria Nigroclavata (Nyl.) Schuler - on bark of Populus tremula; $22\left[\mathrm{R}_{1}\right]$.

\# Cercidospora stenotropae Nav.-Ros. \& Hafellner - on thallus and apothecia of Lecanora polytropa on granite boulders; 12, 26 [R]; 2018 (H). - New to European Russia except Caucasus, known from Republic of Adygeya (Zhurbenko \& Kobzeva, 2016). Distribution in Fennoscandia and Baltic countries: not reported. In Europe known from Austria, Belgium, Germany, Italy, and Spain (Calatayud et al., 2013). This species was provisionally described by Calatayud et al. (2013). The asci are usually 8 -spored in contrast to the 4-spored asci of C. epipolytropa, the second known species growing on taxa of the Lecanora polytropa group (Calatayud et al., 2013).

Cetraria aculeata (Schreb.) Fr. - on sandy soil; $1,3,5,11,12,14,16,23,26,32$ [O].

CETRARIA ERICETORUM Opiz subsp. ERICETORUM - on sandy soil; 5, 8, 14, 16, 18, 26 [R].

Cetraria islandica (L.) Ach. - on soil; 1, 4, 5, $8,12,14,16,18,23,26,32$, a21; subsp. ISLANDICA "f. SOREDIATA (Schaer.) Arnold" - on sandy soil; 14. [O]. On soil in different localities (Liksakova \& Glazkova, 2018).

Cetraria sepincola (Ehrh.) Ach. - on bark of Alnus glutinosa, Juniperus communis; 16, 22, 23 [R].

Cetrariella commixta (Nyl.) A. Thell \& Kärnefelt - on granite boulders; 16, a12 [R]. Red Data Book of LR (Red..., 2018).

ChaEnotheca CHRYsocephala (Turner ex Ach.) Th. Fr. - on bark of Alnus glutinosa, Picea abies, Pinus sylvestris, wood of Picea abies and Pinus sylvestris; 19-21, 24, 28, 30 [R].

Chaenotheca ferruginea (Turner ex Sm.) Mig. on bark of Picea abies, Pinus sylvestris; 21, 24, 27, 28, 30 [R].

Chaenotheca furfuracea (L.) Tibell - on upturned roots, bark of Alnus glutinosa, granite boulders; 20, 22, a18 [R].

Chaenotheca trichialis (Ach.) Th. Fr. - on bark of Picea abies, wood of Picea abies and Pinus sylvestris; 19-21, 27, 28 [R]. 
¿\# Chaenothecopsis nigra Tibell - on thallus of Parmelia sp. on vertical surface of mossy granite boulder; $20\left[\mathrm{R}_{1}\right]$.

+ Chaenothecopsis pusilla (Ach.) A. F. W. Schmidt - on bark of Picea abies; $28\left[\mathrm{R}_{1}\right]$.

Circinaria Contorta (Hoffm.) A. Nordin, S. Savić $\&$ Tibell - on concrete; a $10\left[R_{1}\right]$.

Circinaria GibBosa (Ach.) A. Nordin, S. Savić \& Tibell - on granite boulders; $18\left[\mathrm{R}_{1}\right]$.

Cladonia ARBuscula (Wallr.) Flot. subsp. ARBusCULA - on soil, wood of Pinus sylvestris; 1 , 3-5, 7, 8, 12, 14, 16, 18, 23, 26, 29, 32, a21. BL1 and BL2, on sandy soil (Lemberg, 1935); on soil in different localities (Liksakova \& Glazkova, 2018). Subsp. MiTis (Sandst.) Ruoss - on sandy soil; $1,4,8,32$. [F].

Cladonia BELLIDIFLORA (Ach.) Schaer. - on sandy soil; 3, 4 [R].

Cladonia Borealis S. Stenroos - on sandy soil; $3,8,18$ [R].

Cladonia botrytes (K. G. Hagen) Willd. - on sandy soil, wood of Pinus sylvestris; 4, 12 [R].

Cladonia Cariosa (Ach.) Spreng. - on soil; 1, 3, a17 [R].

Cladonia CaRneola (Fr.) Fr. - on sandy soil; 3, $8,14,16[R]$.

Cladonia Cenotea (Ach.) Schaer. - on soil, bark of Pinus sylvestris, wood; 2-4, 7, 8, 18, 20, 21, 24, 29, 32 [O].

Cladonia CERVICORNis (Ach.) Flot. - on sandy soil; $3,4,8,12,14,18$ [R].

Cladonia chlorophaea (Flörke ex Sommerf.) Spreng. s. str. - on soil, bark of Pinus sylvestris and Quercus robur, 1, 27, 29, 32, a7, a15, a17 [R]. Thalli contain fumarprotocetraric, protocetraric, and fatty acids.

Cladonia Coniocraea (Flörke) Spreng. - on soil, bark of Alnus glutinosa, Betula pubescens, Juniperus communis, Picea abies, Pinus sylvestris, Quercus robur, and wood; 2, 6-9, 19-21, 24, 27, 28, 30, a15 [O].

Cladonia Cornuta (L.) Hoffm. subsp. CORNuTA - on soil, bark of Pinus sylvestris, on wood; 3, 4, 7-9, 14, 18, 20, 21, 24, 29, 32, a21 [O]. BL2, on sandy soil (Lemberg, 1935).

Cladonia CRISPATA (Ach.) Flot. var. CRISPATA - on sandy soil and wood of Pinus sylvestris; 3-5, 8, 14, 18, 23, 29, 32; var. CETRARIFORMIS (Delise) Vain. - on sandy soil; 8, 12, 26. [O].

Cladonia DEFORMis (L.) Hoffm. - on soil and wood; $3,7,8,12,14,18,26,29,32$ [O].
Cladonia digitata (L.) Hoffm. - on bark of Alnus glutinosa, Juniperus communis, Pinus sylvestris, on wood; 2, 9, 20, 21, 24, 30 [R].

Cladonia fimbriata (L.) Fr. - on soil, bark of Picea abies, Pinus sylvestris, on wood of Pinus sylvestris; $2,7,8,12,14,21,26-29$ [O]. Thalli contain fumarprotocetraric acid.

Cladonia Floerkeana (Fr.) Flörke - on sandy soil and wood; 1, 3, 4, 8, 11, 14, 16, 18, 26, $32[\mathrm{O}]$.

Cladonia furcata (Huds.) Schrad. - on soil; 1, 4 , $5,8,12,14,16,18,23,26,29$, a13, a2 1 [O].

Cladonia GRACILIS (L.) Willd. subsp. GRACILIS - on sandy soil; 3, 8, 14, 18, 32; subsp. TURBINATA (Ach.) Ahti - on soil; 4, 7, 8, 14, 18, 29, 32, a21. [O].

Cladonia GRAYI G. Merr. ex Sandst. - on primary soil on granite boulder; $7\left[\mathrm{R}_{1}\right]$. Thallus contains fumarprotocetraric and grayanic acids.

Cladonia Macilenta Hoffm. - on sandy soil and wood; 1, 4, 5, 16, 24, 32 [R].

Cladonia Macrophylla (Schaer.) Stenh. - on sandy soil; $3\left[R_{1}\right]$. Red Data Book of LR (Red..., 2018).

Cladonia novochlorophaEa (Sipman) Brodo et Ahti - on sandy soil; $32\left[\mathrm{R}_{1}\right]$. Thallus contains sekikaic $\&$ homosekikaic acids.

Cladonia OCHROCHLORA Flörke - on sandy soil, bark of Alnus glutinosa, Pinus sylvestris; 4, $21,30[R]$.

Cladonia Phyllophora Hoffm. - on soil; 3-5, 7 , $8,12,14,16,18,26,29,32$ [O]. On soil in different localities (Liksakova \& Glazkova, 2018).

Cladonia Pleurota (Flörke) Schaer. - on sandy soil, bark of Pinus sylvestris, wood; 3, 8, 14, 16, 18, 20, 29, 32 [O].

Cladonia PyXidata (L.) Hoffm. - on sandy soil; 1 , 3, 8, 12, 14, 16, 18, 23, 29 [O].

Cladonia Ramulosa (With.) J. R. Laundon - on sandy soil; 1, 3, 5, 8, 12, 16, 18, 23, 32 [O].

Cladonia Rangiferina (L.) F. H. Wigg. - on soil, wood; $1,3-5,7,8,12,14,16,18,23,26$, 29, 32, a2 1 [F]. BL2, on sandy soil (Lemberg, 1935); on soil in different localities (Liksakova \& Glazkova, 2018).

ClADONIA REI Schaer. - on soil and decaying wood; $1,4,5,8,12,14,16,18,26,29$, a17 [O].

Cladonia scabriuscula (Delise) Nyl. - on sandy soil; 4, 12, a5 [R]. Red Data Book of LR (Red..., 2018).

Cladonia squamosa Hoffm. - on sandy soil; 3, 4, $8,11,12,16,18[\mathrm{O}]$. 
Cladonia stellaris (Opiz) Pouzar \& Vězda - on sandy soil; 3, 8, 12, 14, 32 [R].

Cladonia STYGia (Fr.) Ruoss - on sandy soil; 8 [R $\mathrm{R}_{1}$. Cladonia subulata (L.) F. H. Wigg. - on soil; 1 , $3,4,8,12,14,16,23,26,29,32$, a21 [O]. Thalli contain fumarprotocetraric and protocetraric acids.

Cladonia sulphurina (Michx.) Fr. - on sandy soil and wood of Pinus sylvestris; 3, 4, 7, 8, 14, $18,26[\mathrm{O}]$.

Cladonia symphycarpa (Flörke) Fr. - on sandy soil; 3, 29, a8 [R].

Cladonia TURGIDA Hoffm. - on sandy soil; a13 [R $\mathrm{R}_{1}$.

Cladonia uncialis (L.) F. H. Wigg. subsp. uncialis - on sandy soil; 3-5, 8, 14, 16, 18, 26, 32; subsp. BIUNCIALIS (Hoffm.) M. Choisy - on sandy soil; 3, 8. [O]. On soil in different localities (Liksakova \& Glazkova, 2018).

Cladonia verticillata (Hoffm.) Schaer. - on soil; $3,5,7,8,14,18,29,32$ [O]. On soil in different localities (Liksakova \& Glazkova, 2018).

Clauzadea monticola (Schaer.) Hafellner \& Bellem. - on concrete; a16 [ $\left.\mathrm{R}_{1}\right]$.

Cliostomum griffithiI (Sm.) Coppins - on bark of Alnus glutinosa, Picea abies, Salix caprea L., Sorbus aucuparia, on wood of Pinus sylvestris; 27, 30, a20 [R].

\# Clypeococcum hypocenomycis D. Hawksw. - on thalli of Hypocenomyce scalaris on bark and wood of Pinus sylvestris and Juniperus communis; 3, 7, 9, 20, 24 [R].

Coenogonium Pineti (Ach.) Lücking \& Lumbsch - on bark of Alnus glutinosa, Betula pubescens, Picea abies, Pinus sylvestris, on wood of Picea abies; 6, 19, 21, 22, 27, 28, 30 [O].

\# Endococcus exerRans Nyl. - on thalli of Rhizocarpon distinctum and $R$. richardii on granite boulders; 1, 11, 15, 23 [R]; det. W. von Brackel, 2018 (H). - New to Russia. Distribution in Fennoscandia and Baltic countries: Sweden (Nordin et al., 2011), Estonia (Randlane et al., 2018). This lichenicolous fungus differs from the two other species growing on Rhizocarpon (E. fusiger and $E$. macrosporus) by narrower spores (Schiefelbein et al., 2017).

\# ERYTHRicium Aurantiacum (Lasch) D. Hawksw. $\&$ A. Henrici - on thallus of Physcia aipolia on bark of Acer platanoides; $25\left[\mathrm{R}_{1}\right]$ (BILAS). - New to European Russia except Caucasus, known from Republic of Karachaevo-Cherkessiya (Zhurbenko \& Kobzeva, 2014). Distribution in Fennoscan- dia and Baltic countries: Sweden (Nordin et al., 2011), Estonia (Randlane et al., 2018), Latvia (Motiejūnaitè et al., 2016), Lithuania (Motiejūnaite, 2017). This fairly common, predominantly physciicolous basidiomycete is only very rarely found with basidiomata. Usually (as in our specimen) it occurs as pale pink 'sporodochia' of 100-150 $\mu \mathrm{m}$ diam. consisting of elongated and single obpyriform cells which are immature basidia and basidiospores (Diederich et al., 2003).

EvERNIA MESOMORPHA Nyl. - on bark of Pinus sylvestris; a13 $\left[\mathrm{R}_{1}\right]$.

EVERNIA PRUNASTRI (L.) Ach. - on bark of Alnus glutinosa, Picea abies, Pinus sylvestris, Sorbus aucuparia; 4, 6, 14, 19, 22, 27, 30 [O].

Flavoplaca Citrina (Hoffm.) Arup, Frödén \& Søchting - on concrete; $26\left[\mathrm{R}_{1}\right]$.

FlaVoplaca DichroA (Arup) Arup, Frödén \& Søchting - on concrete; a10 $\left[\mathrm{R}_{1}\right](\mathrm{H})$. - New to WLR, previously known from SPb (Vondrák et al., 2010).

Flavoplaca Limonia (Nimis \& Poelt) Arup, Frödén \& Søchting - on concrete; $17\left[\mathrm{R}_{1}\right]$ (H, LECB). - New to European Russia except Caucasus, previously known from Krasnodar Territory (Vondrák et al., 2009). Distribution in Fennoscandia and Baltic countries: Sweden (Nordin et al., 2011). F. limonia belongs to the $F$. citrina group. It has a dull, greenish yellow to bright yellow, often white-pruinose thallus and forms a continuous areolate crust. $F$. citrina is very similar but $F$. limonia is usually recognized by the presence of larger diaspores that are blastidia, rather than soredia (Arup, 2011). The species grows often under rather shaded conditions near the ground (Arup, 2011; Wirth et al., 2013). In Germany, it prefers eutrophicated artificial walls fertilized by urine (Wirth et al., 2013; Schiefelbein et al., 2014).

FlaVoplaCa MARINA (Wedd.) Arup, Frödén \& Søchting - on granite boulders; 1,15 [R].

Flavoplaca OASIS (A. Massal.) Arup, Frödén \& Søchting - on concrete; 26, a10 [R] (H). New to North-Western European Russia. In European Russia is known from Moscow Region (Muchnik, 2016). Distribution in Fennoscandia and Baltic countries: Norway, Sweden (Nordin et al., 2011). Similar to the Athallia holocarpa group. In the past, it is often recorded under the name Caloplaca holocarpa (Hoffm. ex. Ach.) A. E. 
Wade, but $F$. oasis has smaller apothecia, smaller spore septa and grows on mortar and concrete (Arup, 2009). In contrast, A. holocarpa and A. vitellinula (Nyl.) Arup, Frödén \& Søchting, another very similar species, inhabit siliceous rocks. Here it is reported also from neighboring Bol'shoy Tuters Island (former Tytärsaari): S shore, E of cape Lommosniemi, old Finnish village, $59^{\circ} 50^{\prime} 10^{\prime \prime} \mathrm{N}, 27^{\circ} 11^{\prime} 56^{\prime \prime} \mathrm{E}$, wasteland, on concrete, 29.05.2015, leg. IS (H).

Fuscidea ARBORICOLA Coppins \& Tønsberg - on bark of Alnus glutinosa, Pinus sylvestris, Quercus robur, Sorbus aucuparia, wood; 6, 21, 22, 27, a15 [R].

FUSCIDEA PUSILLA Tønsberg - on bark of Betula pubescens, Juniperus communis, Picea abies, Pinus sylvestris, wood; 2, 4, 6, 7, 9, 19-21, 24, 27, 28, 30 [O].

GRAPHIS SCRIPTA (L.) Ach. - on bark of Alnus glutinosa; $30\left[\mathrm{R}_{1}\right]$.

GyaleCtA FAgicola (Hepp ex Arnold) Kremp. - on bark of Acer platanoides, Populus tremula; $22,25[R]$.

Gyalolechia flavorubescens (Huds.) Søchting, Frödén \& Arup - on bark of Populus tremula; $9\left[\mathrm{R}_{1}\right]$.

HAEMATOMMA OCHROLEUCUM (Neck.) J. R. Laundon - on granite boulder; $20\left[R_{1}\right]$. Thallus contains atranorin, zeorin, usnic, porphyrilic and fatty acids.

\# Homostegia Piggotil (Berk. \& Broome) P. Karst. - on thalli of Parmelia saxatilis on granite boulders; 23, 31, a4 [R].

HYdROPUnCTARIA MAURA (Wahlenb.) Keller, Gueidan \& Thüs - on granite boulders; 1, 10, $13,15,31[R]$.

HYPOCENOMYCE SCALARIS (Ach.) M. Choisy - on bark of Juniperus communis, Picea abies, Pinus sylvestris, on wood; 2, 3, 7, 9, 16, 18-21, 23, 24, 28, 32 [F].

HYPOGYMNIA FARINACEA Zopf - on bark of Pinus sylvestris; 14, 23, 24 [R].

HyPogymnia PHYSODEs (L.) Nyl. - on bark of Acer platanoides, Alnus glutinosa, Betula pubescens, Juniperus communis, Malus domestica Borkh., Picea abies, Pinus sylvestris, Populus tremula, Quercus robur and Sorbus aucuparia, on wood, granite boulders, once on sandy soil; $2,4,6,7,9,13,14,16,18-28$, 30-32, a7, a9, a15 [C]. Coll. EN: on bark of Pinus sylvestris, 1851 (H 8005054); coll. NB: on bark of tree, 1993 (Alexeeva, 2005).
Hypogymnia tubulosa (Schaer.) Hav. - on bark of Alnus glutinosa, Juniperus communis, Picea abies, Pinus sylvestris, Populus tremula, Sorbus aucuparia, on wood; 2, 4, 7, 9, 14, 19-23, 26, 28, 30, 31, a7, a9 [F].

\# Illosporiopsis Christiansenil (B. L. Brady \& D. Hawksw.) D. Hawksw. - on thalli of Physcia spp. on bark of Acer platanoides and Sorbus aucuparia; 16, 25 [R].

\# Intralichen Christiansenil (D. Hawksw.) D. Hawksw. \& M. S. Cole on Myriolecis dispersa growing on concrete; a $10\left[\mathrm{R}_{1}\right]$. New to North-Western European Russia, the nearest locality in Russia belongs to Samara Region (Tsurykau \& Korchikov, 2017). Distribution in Fennoscandia and Baltic countries: Norway, Sweden, Finland (Nordin et al., 2011), Estonia (Randlane et al., 2018), Latvia (Motiejūnaitè et al., 2016), Lithuania (Motiejūnaite, 2017). The species is characterized by hyaline to pale brown immersed conidiophores, and conidia pale brown, ellipsoid, with rounded ends, 1-septate, 5-8(-9) × 4-6 $\mu \mathrm{m}$ (Hawksworth, 1983).

IMSHAUGIA ALEURITES (Ach.) S. L. F. Meyer - on bark of Pinus sylvestris, on wood; 3, 15, $16,24[\mathrm{R}]$.

LAMBIELla FURVELLA (Nyl. ex Mudd) M. Westb. \& Resl - on granite boulders; $18\left[\mathrm{R}_{1}\right]$.

LeCANiA CYRTELla (Ach.) Th. Fr. - on bark of Acer platanoides, Populus tremula; 25, a20 [R].

LeCANia NAEGELII (Hepp) Diederich \& van den Boom - on bark of Acer platanoides, Populus tremula, Sorbus aucuparia, on wood; 9, 22, 25, a17 [R].

LeCANIA SYlVestRis (Arnold) Arnold - on bones; 13, a3, a12 [R].

LeCANORA Aitema (Ach.) Hepp - on bark of Pinus sylvestris, on wood; 4, 13, a9 [R].

LeCANORA AlBellula (Nyl.) Th. Fr. - on bark of Juniperus communis, Pinus sylvestris, Syringa vulgaris L., on wood; 4, 15, 16, 18, 25, 32, a9 [R].

LECANORA ALlOPHANA Nyl. - on bark of Populus tremula; 9, 22 [R].

LECANORA CADUBRIAE (A. Massal.) Hedl. - on bark of Pinus sylvestris; $7\left[\mathrm{R}_{1}\right]$.

LECANORA CARPINEA (L.) Vain. - on bark of Acer platanoides, Alnus glutinosa, Juniperus communis, Malus domestica, Pinus sylvestris, Populus tremula, Rosa rugosa Thunb., Sorbus aucuparia, Syringa vulgaris, on wood; $6,9,13,16,22,23,25,27,30$, a7, a9 [O]. 
Coll. NB: on bark of deciduous tree, 1993 (Alexeeva, 2005).

LECANORA CHLAROTERA Nyl. - on bark of Acer platanoides, Sorbus aucuparia, on wood; 22, 25, a9 [R].

LECANORA CIRCUMBOREALIS Brodo \& Vitik. - on bark of Picea abies, Pinus sylvestris, on wood; 4, 27, a9 [R].

LECANORA EXPALLENS Ach. - on bark of Alnus glutinosa, Betula pubescens, Picea abies, Quercus robur, on wood of Pinus sylvestris; $6,22,27$, a 15 [R]. Thalli contain usnic and thiophanic acids, zeorin, cf. arthothelin and unidentified xanthone called 'expallens unknown'.

LECANORA HELICOPIS (Wahlenb.) Ach. - on granite boulders; 1, 10, 15, 31 [R].

LECANORA HYPOPTELLA (Nyl.) Grummann - on bark of Pinus sylvestris; 7, 20 [R].

LECANORA INTRICATA (Ach.) Ach. - on granite boulders, on bricks; $7,11,12,15,16,18,23$, $25,26,29,31$, a16 [O].

LECANORA MUGHICOLA Nyl. - on wood; 13 [R $]$.

LECANORA PHAEOSTIGMA (Körb.) Almb. - on bark of Pinus sylvestris; $4\left[\mathrm{R}_{1}\right]$.

LECANORA POLYTROPA (Ehrh. ex Hoffm.) Rabenh. - on wood, granite boulders and small pebbles, iron, leather; $7,8,11-13,15,16,18$, 23, 26, 29, 31, a1, a9, a14 [O].

LECANORA PULICARIS (Pers.) Ach. - on bark of Alnus glutinosa, Juniperus communis, Pinus sylvestris, Quercus robur, Sorbus aucuparia, on wood; $6,13,14,16,20-22,27,30$, a9, a15 [O].

LECANORA RIMICOLA H. Magn. - on granite boulders; $1\left[R_{1}\right]$.

LECANORA RUPICOLA (L.) Zahlbr. - on granite boulders; 15, 23 [R].

LECANORA SALIGNA (Schrad.) Zahlbr. - on wood; a9 $\left[R_{1}\right]$.

LECANORA SUBINTRICATA (Nyl.) Th. Fr. - on wood; 13, 26, a9 [R].

LECANORA SYMMICTA (Ach.) Ach. - on bark of Acer platanoides, Alnus glutinosa, Juniperus communis, Pinus sylvestris, Quercus robur, Syringa vulgaris, Sorbus aucuparia, on wood; 1 , 2, 6, 15, 16, 25, 27, 31, a6, a7, a9, a15 [O]. Coll. NB: on bark of deciduous tree, 1993 (Alexeeva, 2005).

LECANORA UMBrina (Ach.) A. Massal. - on bark of Alnus glutinosa, Pinus sylvestris, Populus tremula, Rosa rugosa, Sorbus aucuparia, on wood, bones, iron; 1, 13, 16, 22, 23, 25, a3, a7, a9, a12, a14, a17 [R].
LECANORA VARIA (Hoffm.) Ach. - on bark of Alnus glutinosa, on wood; 3, 13, 16, 26, a7, a9 [R].

LECIDEA AURICUlata Th. Fr. subsp. AuRICUlata - on granite boulders; 7, 8 [R] (LECB). New to LR. Distribution in North-Western European Russia outside of LR: Republic of Karelia (Fadeeva et al., 2007). Distribution in Fennoscandia and Baltic countries: Norway, Sweden, Finland (Nordin et al., 2011). Characterized by the raised, thick, internally reddish flush and $\mathrm{C}$ - exciple, as well as the narrow ellipsoid ascospores (Smith et al., 2009).

LECIDEA HAERJEDALICA H. Magn. - on granite boulder in supralittoral; $15\left[\mathrm{R}_{1}\right]$. New to European Russia, in Russia known from Irkutsk Region (Makryi, 2005). Distribution in Fennoscandia and Baltic countries: Norway, Sweden (Nordin et al., 2011). Our specimen conforms to the protologue (Magnusson, 1948) and is characterized by very poorly developed dark greyish thallus which is almost totally absent (poorly visible in cracks in stone only) and multiple irregular-shaped small, 0.2-0.3(0.5) $\mathrm{mm}$, black sessile apothecia with thick convex margin. Epihymenium greenish-black with uneven rough surface, hymenium hyaline 45-65 $\mu \mathrm{m}$, exciple well-developed, greenishbrownish-violet, dark blackish outside, paler inside, $\mathrm{J}+$ violet. Hypothecium pale with a shade of violet, which is more visible in $\mathrm{K}$. Spores hyaline, broad ellipsoid, 11-13 $\times$ 4.5-6.5 $\mu \mathrm{m}$.

LECIDEA LAPICIDA (Ach.) Ach. var. LAPICIDA - on granite boulders; 15, 18, 31; var. PANTHERINA Ach. - on granite boulders; 11, 12, 15, 18, 23, 31. [R].

LECIDEA NYLANDERI (Anzi) Th. Fr. - on bark of Alnus glutinosa, Picea abies, Pinus sylvestris; 2, 6, 7, 20, 21, 24, 27, 28, 30 [O].

LeCidea Plebeja Nyl. - on wood of Pinus sylvestris; $3\left[\mathrm{R}_{1}\right]$.

LECIDEA TURGIDULA Fr. - on bark of Alnus glutinosa, Pinus sylvestris, on wood of Picea abies; 7, 19-21, 28, 30 [R].

LeCidella elaeochroma (Ach.) M. Choisy - on bark of Acer platanoides, Alnus glutinosa, Juniperus communis, Populus tremula, Sorbus aucuparia, on wood; 1, 9, 13, 16, 22, 25, 27, a7 [O].

LECIDELla EUPHOREA (Flörke) Hertel - on bark of Populus tremula; $9\left[\mathrm{R}_{1}\right]$. 
LECIDELLA MEIOCOCCA (Nyl.) Leuckert \& Hertel - on granite boulders; $11\left[\mathrm{R}_{1}\right]$.

Lecidella stigmatea (Ach.) Hertel \& Leuckert on brick, concrete, slate, bones; 26, 29, a3, a10, a19, a21 [R].

LEPRA AMARA (Ach.) Hafellner - on bark of Alnus glutinosa; $30\left[\mathrm{R}_{1}\right]$.

LEPRARIA ELOBATA Tønsberg - on bark of Alnus glutinosa, Betula pubescens, Picea abies, Pinus sylvestris, Quercus robur, Sorbus aucuparia, on wood and granite boulders; $2,7,9,19,21,22,24,27,28,30, a 2, a 15$, a16 [O]. Thalli contain atranorin, zeorin, and stictic acid complex.

LEPRARIA INCANA (L.) Ach. - on primary soil on granite boulder, bark of Alnus glutinosa, Betula pubescens, Juniperus communis, Picea abies, Pinus sylvestris, Sorbus aucuparia, on wood of Picea abies and granite boulders; $6,19,20,22,24,28,30$ [O]. Thalli contain atranorin, zeorin, and divaricatic acid.

LEPRARIA JACKII Tønsberg s. 1. - on bark of Picea abies, Pinus sylvestris, on wood of Picea abies and granite boulders; 1, 2, 19-21, 28, a2 [R]. Thalli contain atranorin, jackinic/ rangiformic, norjackinic/norrangiformic, and roccellic acids.

LEPRARIA LOBIFICANS Nyl. - on bark of Quercus robur, a $15\left[\mathrm{R}_{1}\right]$.

LEPTORHAPHIS ATOMARIA (Ach.) Szatala - on bark of Populus tremula; 22, a20 [R].

\# Lichenoconium erodens M. S. Christ. \& D. Hawksw. - on thallus of Parmelia sulcata on bark of Juniperus communis; 16 [ $\left.\mathrm{R}_{1}\right]$.

\# Lichenoconium lecanorae (Jaap) D. Hawksw. on apothecia of Lecanora carpinea on bark of Sorbus aucuparia; $22\left[\mathrm{R}_{1}\right]$.

\# Lichenoconium usneae (Anzi) D. Hawksw. - on thallus of Ramalina subfarinacea on granite boulder; $31\left[\mathrm{R}_{1}\right]$.

\# Lichenoconium xanthoriae M. S. Christ. - on apothecia of Polycauliona polycarpa on granite boulders on seashore; $31\left[R_{1}\right]$.

\# Lichenopeltella coppinsi Earl.-Benn. \& D. Hawksw. - on thallus of Verrucaria muralis on brick; $29\left[\mathrm{R}_{1}\right]$; conf. W. von Brackel, 2018 (H). - New to Russia. Distribution in Fennoscandia and Baltic countries: Estonia (Randlane et al., 2018). Only L. coppinsii and L. hydrophila have previously been reported from Verrucaria thalli. They differ in the width of spores and asci. The spores of $L$. hydrophila are broader and the asci smaller (Earland-Bennett \& Hawksworth, 1999).

\# Lichenosticta AlCicoRnaria (Linds.) D. Hawksw. - on thalli of Cladonia chlorophaea s. 1. on soil; 23, 26 [R].

\# Lichenostigma MAURERI Hafellner - on thallus of Usnea hirta on twig of Juniperus communis; $24\left[\mathrm{R}_{1}\right]$.

Melanelia StYgia (L.) Essl. - on granite boulders; $7\left[R_{1}\right]$. Red Data Book of LR (Red..., 2018).

MelaneliXia fuliginosa (Fr. ex Duby) O. Blanco et al. - on granite boulders; 1, 15, 23, 31, a4 [R].

MelaneliXia subaurifera (Nyl.) O. Blanco et al. - on bark of Juniperus communis, Populus tremula, Sorbus aucuparia, on wood of Pinus sylvestris; 16, 22, 27 [R].

Melanohalea exasperata (De Not.) O. Blanco et al. - on bark of Acer platanoides; $25\left[\mathrm{R}_{1}\right]$.

Melanohalea exasperatula (Nyl.) O. Blanco et al. - on bark of Acer platanoides, Alnus glutinosa, Picea abies, Pinus sylvestris, Sorbus aucuparia, on wood and granite boulders; $2,4,14,16,19,22-25,27,28,30,31$, a7, a9, a12 [F].

Melanohalea Olivacea (L.) O. Blanco et al. - on bark of Alnus glutinosa, Populus tremula, Sorbus aucuparia, on wood; 9, 16, 22, a7, a9 [R].

Micarea byssacea (Th. Fr.) Czarnota et al. - on bark of Alnus glutinosa, Picea abies, on wood of Pinus sylvestris; 6, 21, 22, 27, 30 [R]. Thalli contain methoxymicareic acid. Det. S. Chesnokov \& L. Konoreva, 2018 and 2019.

Micarea DEnigrata (Fr.) Hedl. - on bark of Alnus glutinosa, Pinus sylvestris, on wood of Pinus sylvestris; 2, 6, 7, 18, 24 [R]. Thalli contain gyrophoric acid. Det. Chesnokov \& Konoreva, 2018 and 2019.

Micarea ERRATICA (Körb.) Hertel, Rambold \& Pietschm. - on small granite pebbles; 12, a17 [R].

Micarea melaena (Nyl.) Hedl. - on bark and wood of Pinus sylvestris; 7, 21 [R].

Micarea Micrococca (Körb.) Gams ex Coppins on bark of Pinus sylvestris, on wood of Picea abies; 19, 27 [R]. Thalli contain methoxymicareic acid. Det. Chesnokov, 2019.

Micarea Misella (Nyl.) Hedl. - on bark of Pinus sylvestris, wood of Picea abies and Pinus sylvestris; 2, 27, 28 [R]. No substances detected. Det. Chesnokov \& Konoreva, 2018. 
Micarea PEliocarpa (Anzi) Coppins \& R. Sant. - on bark of Pinus sylvestris; $2\left[\mathrm{R}_{1}\right]$. Thallus contains gyrophoric acid. Det. Konoreva, 2018.

MicAREA PRASINA Fr. s. str. - on wood of Picea abies; $30\left[\mathrm{R}_{1}\right]$. Thallus contains micareic acid. Det. Chesnokov, 2019.

\# Monodictys epilepraria Kukwa \& Diederich - on thallus of Lepraria jackii on granite boulder; $1\left[\mathrm{R}_{1}\right]$.

MonTANELIA DISJUnCtA (Erichsen) Divakar et al. on granite boulders; 7,15 [R].

MonTANELIA SOREdiATA (Ach.) Divakar et al. - on granite boulders; 7, 15, 16, 23 [R].

\# Muellerella Pygmaea (Körb.) D. Hawksw. - on thallus of Lecidea lapicida var. pantherina on granite boulder on seashore; $31\left[R_{1}\right]$.

MycoBILIMBIA EPIXANTHOIDES (Nyl.) Vitik. et al. on bark and mosses on bark of Acer platanoides; $25\left[\mathrm{R}_{1}\right]$.

Mycoblastus alpinus (Fr.) Th. Fr. ex Hellb. - on mosses on granite boulder; $20\left[\mathrm{R}_{1}\right]$.

+ Mrcocalicium subtile (Pers.) Szatala - on wood of Pinus sylvestris; 18, 20, 24 [R].

Myriolecis ALBESCENS (Hoffm.) Śliwa et al. - on granite boulders and concrete; 17 , a10 [R].

Myriolecis CREnUlata (Hook.) Śliwa et al. - on bones; a3 [ $\left.\mathrm{R}_{1}\right]$.

MYRIOLECIS DISPERSA (Pers.) Śliwa et al. - on granite boulders, concrete, bones; 17, 26, a10, a12, a21 [R].

Myriolecis HAgEni (Ach.) Śliwa et al. - on bark of Juniperus communis, Populus tremula, on wood and bones; 9, 13, 16, a3, a9, a12 [R].

MyRIOLECIS PERPRUINOSA (Fröberg) Śliwa et al. - on concrete; $26\left[R_{1}\right]$. - New to WLR, previously known from ELR (Kuznetsova et al., 2016).

Myriolecis Salina (H. Magn.) Śliwa et al. s. 1. - on granite boulders and concrete; 1, 10, 13, 15, 17,31 , a $10[\mathrm{R}]$.

Myriolecis SEMiPAlLida (H. Magn.) Śliwa et al. on concrete, slate, and bones; 26, a3, a10, a19 [R].

Myriolecis zosterae (Ach.) Śliwa et al. - on wood and bones; 13, a3, a9, a12, a17 [R] (LECB). New to North-Western European Russia, the nearest locality in Russia belongs to Murmansk Region (Urbanavichus et al., 2008). Distribution in Fennoscandia and Baltic countries: Norway (Øvstedal et al., 2009), Sweden (Śliwa, 2007), Estonia (Randlane et al., 2018). Closely related to $M$. hagenii, but differs by the larger apothecia, sinuous and concave mature apothecial disc, normally without of pruina, and not at all granular epithecium (Sliwa, 2007).

MYRIOSPORA MYOCHROA (M. Westb.) K. Knudsen \& L. Arcadia - on granite boulders; $31\left[\mathrm{R}_{1}\right](\mathrm{H}$, LECB). - New to European Russia, known from Krasnoyarsk Territory (Zhdanov, 2013). Distribution in Fennoscandia and Baltic countries: Norway, Sweden, Finland (Nordin et al., 2011). On coastal rocks and boulders of the Baltic Sea, four Myriospora species can be found (Westberg et al., 2010, Schiefelbein et al., 2015, Knudsen et al., 2017). Characteristic features of $M$. myochroa are crystals in the cortex (norstictic acid), visible in polarized light/phase contrast (section) and a very inconsistent reaction with potassium hydroxide. The presence of crystals in the cortex distinguishes it from $M$. rhagadiza (Nyl.) K. Knudsen \& L. Arcadia and $M$. rufescens (Ach.) Hepp ex Uloth, and the weak or no reaction with $\mathrm{KOH}$ from $M$. smaragdula (Wahlenb. ex Ach.) Nägeli ex Uloth (Westberg et al., 2010).

NAETRocymbe Punctiformis (Pers.) R. C. Harris - on bark of Alnus glutinosa, Sorbus aucuparia; 27, a7 [R].

\# Nectriopsis lecanodes (Ces.) Diederich \& Schroers - on thallus of Peltigera polydactylon on sandy soil; a21 $\left[R_{1}\right]$. - New to WLR, previously known from ELR (Zhurbenko, 2009).

\# Nesolechia fusca (Triebel \& Rambold) PérezOrtega - on thallus of Xanthoparmelia conspersa on granite boulder; 25 [R $\mathrm{R}_{1}$ (BILAS). - New to North-Western European Russia. In European Russia known from Ural part of Yamal-Nenets Autonomous Area (Zhurbenko, 2008). Distribution in Fennoscandia and Baltic countries: Sweden (Nordin et al., 2011), Estonia (Randlane et al., 2018). Characterized by dark brown hypothecium, blackish ascomata (when very young) and inhabiting different hosts of Parmeliaceae than Nesolechia oxyspora (Tul.) A. Massal. var. oxyspora, mainly Xanthoparmelia species (Triebel et al., 1995; Diederich, 2003).

OCHROLECHIA ARBOREA (Kreyer) Almb. - on bark of Alnus glutinosa; $6\left[\mathrm{R}_{1}\right]$. Thallus contains gyrophoric, lecanoric acids, and lichexanthone.

OCHROLECHIA BAHUSIENSIS H. Magn. - on bark of Alnus glutinosa, Quercus robur; 22, 30, a15 [R]. Thalli contain lecanoric, gyrophoric acids and murolic acid complex. 
OCHROLECHIA MICROSTICTOIDES Räsänen - on bark of Pinus sylvestris, on bark and wood of Picea abies; 21, 27 [R]. Thalli contain variolaric, lichesterinic acids, and substances called 'microstictoides unknowns' (see Kukwa, 2011).

OPEGRAPHA VULGATA (Ach.) Ach. - on bark of Salix caprea; a20 $\left[\mathrm{R}_{1}\right]$.

PARMELIA ERnStiae Feuerer \& A. Thell - on bark of Quercus robur; a15 [ $\left.\mathrm{R}_{1}\right]$.

PARMELIA SAXATILIS (L.) Ach. - on bark of Juniperus communis, Pinus sylvestris, on wood and granite boulders; $1,7,12,15,16,18,20$, 23-26, 31, a4, a6, a16 [O].

PARMELIA SUlCATA Taylor - on bark of Acer platanoides, Alnus glutinosa, Juniperus communis, Picea abies, Pinus sylvestris, Populus tremula, Quercus robur, Sorbus aucuparia, on wood and granite boulders; 2, 4, 6, 9, 12, 13, 15, 16, 18, 19, 22-25, 27, 30, a2, a6, a7, a9, a15 [F]. Coll. NB: on bark of deciduous tree, 1993 (Alexeeva, 2005).

PARMELIOPSIS AMBIGUA (Wulfen) Nyl. - on bark of Alnus glutinosa, Betula pubescens, Juniperus communis, Picea abies, Pinus sylvestris, Sorbus aucuparia, on wood; 4, 6, 7, 9, 11, 12, 16, 18-24, 27, 28, 30, 32 [F]. Coll. NB: on bark of coniferous tree, 1993 (Alexeeva, 2005).

PARMELIOPSIS HYPEROPTA (Ach.) Arnold - on bark of Betula pubescens, Juniperus communis, Pinus sylvestris, on wood; 11, 19, 20, 23 [R].

Peltigera canina (L.) Willd. - on sandy soil, on mosses on granite boulder; 25, 29 [R].

Peltigera Didactyla (With.) J. R. Laundon - on soil; 1, a17, a19, a21 [R].

Peltigera extenuata (Nyl. ex Vain.) Lojka - on sandy soil; $29\left[\mathrm{R}_{1}\right]$.

Peltigera NeCKeri Hepp ex Müll. Arg. - on sandy soil; $26\left[\mathrm{R}_{1}\right]$.

Peltigera neopolydactyla (Gyeln.) Gyeln. - on sandy soil; a13 $\left[R_{1}\right]$.

Peltigera POlydactylon (Neck.) Hoffm. - on soil; 26, 29, a13, a17, a21 [R].

Peltigera Rufescens (Weiss) Humb. - on soil and mosses; 12, 16, 26, a1 [R].

PERTUSARIA PUPILlaris (Nyl.) Th. Fr. - on bark of Sorbus aucuparia; $27\left[\mathrm{R}_{1}\right]$.

PhaEophyscia CILIATA (Hoffm.) Moberg - on bark of Populus tremula; 9, 22 [R].

PhaEophyscia Endophoenicea (Harm.) Moberg - on bark of Populus tremula; $22\left[\mathrm{R}_{1}\right]$. Red Data Book of LR (Red..., 2018).
Phaeophyscia nigricans (Flörke) Moberg - on concrete; a10, a21 [R].

PhAEOPHYSCIA ORBICULARIS (Neck.) Moberg - on bark of Populus tremula, granite boulders, concrete, and bones; 9, 17, 26, a3, a10, a21 [R].

PhaEOPHYSCIA SCIASTRA (Ach.) Moberg - on concrete; a $10\left[R_{1}\right]$.

Phlyctis ARgena (Spreng.) Flot. - on bark of Alnus glutinosa, Betula pubescens, Populus tremula, Quercus robur, Sorbus aucuparia; 6, 9, 22, 30, a $15[R]$.

Physcia ADSCEndens H. Olivier - on bark of Acer platanoides, Alnus glutinosa, Juniperus communis, Populus tremula, Sorbus aucuparia, on concrete and bones; 9, 16, 22, 23, 25, a3, a7, a10, a21 [R]. Coll. NB: on bark of deciduous tree, 1993 (Alexeeva, 2005).

Physcia Aipolia (Ehrh. ex Humb.) Fürnr. - on bark of Acer platanoides, Alnus glutinosa, Juniperus communis, Malus domestica, Pinus sylvestris, Populus tremula, Syringa vulgaris, Sorbus aucuparia, on wood and bones; 9, 16, 22, 23, 25, a3, a7, a9, a12 [R].

Physcia ALNOPHILA (Vain.) Loht. et al. - on bark of Populus tremula, Sorbus aucuparia; 9, 16 [R].

PhysCiA CAESIA (Hoffm.) Fürnr. - on granite boulders, concrete, iron; 1, 10, 13, 15, 17, 26, 31 , a10, a2 1 [O].

Physcia DUBia (Hoffm.) Lettau - on bark of Sorbus aucuparia, on wood, granite boulders, concrete, slate, bones; 1, 10, 13, 15-17, 26, a3, a6, a16, a19 [O].

PHYSCIA STELLARIS (L.) Nyl. - on bark of Acer platanoides, Alnus glutinosa, Rosa rugosa, Sorbus aucuparia; 16, 25, a7 [R]. Coll. NB: on bark of deciduous tree, 1993 (Alexeeva, 2005).

Physcia tenella (Scop.) DC. - on bark of Acer platanoides, Juniperus communis, Malus domestica, Picea abies, Populus tremula, Sorbus aucuparia, on wood, granite boulders, concrete, bones; 1, 9, 13, 15-17, 22, 25, 26, 28, 31, 32, a9, a10, a12 [O]. Coll. NB: on bark of deciduous tree, 1993 (Alexeeva, 2005).

PhysCONIA DISTORTA (With.) J. R. Laundon - on bark of Populus tremula; $9\left[\mathrm{R}_{1}\right]$.

Placynthiella dasaea (Stirt.) Tønsberg - on bark of Picea abies, wood, mosses, and plant debris; 2, 7, 9, 18, 19, 24, 27-30 [O].

Placynthiella icmalea (Ach.) Coppins \& P. James - on sandy soil, wood, rubber; $1,2,4,8,9$, 16, 24, 27, 29, 31, 32, a7 [O]. 
Placynthiella oligotropha (J. R. Laundon) Coppins \& P. James - on sandy soil; $1,3-5,8$, $14,16,18,32$ [O].

Placynthiella Uliginosa (Schrad.) Coppins \& P. James - on sandy soil, bark of Juniperus communis, wood of Pinus sylvestris; 3-5, 8, $14,16,18,24,26,29,32$ [O].

Placynthium NigRum (Huds.) Gray - on slate; a19 $\left[R_{1}\right]$.

Platismatia glauca (L.) W. L. Culb. \& C. F. Culb. - on bark of Alnus glutinosa, Juniperus communis, Picea abies, Pinus sylvestris, Sorbus aucuparia, on wood of Picea abies and Pinus sylvestris; 2, 6, 7, 14, 18, 19, 21-23, 28, $30[\mathrm{O}]$.

Polycauliona candelaria (L.) Frödén, Arup \& Søchting - on wood and granite boulders; 1, 10, 13, 15-17, a9 [R].

Polycauliona Phlogina (Ach.) Arup, Frödén \& Søchting - on wood; 13, a9, a17 [R]. - New to WLR, previously known from $\mathrm{SPb}$ and ELR (Vondrák et al., 2010).

PolyCAUliona POLYCARPa (Hoffm.) Frödén, Arup \& Søchting - on bark of Acer platanoides, Alnus glutinosa, Juniperus communis, Malus domestica, Pinus sylvestris, Populus tremula, Rosa rugosa, Sorbus aucuparia, on wood, granite boulders, bones, iron; 1, 4, 9, 13-16, $22,23,25,27,31,32, a 3, a 6, a 7$, a9 $[\mathrm{F}]$. Coll. NB: on bark of deciduous tree, 1993 (Alexeeva, 2005).

\# Polycoccum Pulvinatum (Eitner) R. Sant. - on thallus of Physcia caesia on granite boulder on seashore; $15\left[\mathrm{R}_{1}\right]$.

PorPidia CRUSTUlata (Ach.) Hertel \& Knoph - on granite boulders and small pebbles, on iron; 7, 29, a17 [R].

PorPIDIA MACROCARPA (DC.) Hertel \& A. J. Schwab - on granite boulders; $7,8[\mathrm{R}]$.

PoRPIDIA SOREDIZODEs (Lamy ex Nyl.) J. R. Laundon - on granite boulders and brick; 7, 25, 29, a16 [R].

PoRPidia TUBERCUlOSA (Sm.) Hertel $\&$ Knoph - on granite boulders; 7, a2, a16 [R]. Thalli contain confluentic and 2'-O-methylperlatolic acids.

\# Pronectria Robergei (Mont. \& Desm.) Lowen on thallus of Peltigera polydactylon on sandy soil; a2 $1\left[\mathrm{R}_{1}\right]$. Anamorph.

PROTOPARMELIOPSIS MURALIS (Schreb.) M. Choisy on wood, granite boulders, concrete; 1, 12, $13,15,16,25,26$, a10 [O].

PROTOTHELENElla CoRRosa (Körb.) H. Mayrhofer \& Poelt - on granite boulders; $7\left[\mathrm{R}_{1}\right]$.
Pseudevernia furfuracea (L.) Zopf - on bark of Alnus glutinosa, Juniperus communis, Picea abies, Pinus sylvestris, Sorbus aucuparia, on wood and granite boulders; 2-4, 14, 16, 18-22, 24, 26-28, 30, 31 [F].

Pseudosagedia Aenea (Wallr.) Hafellner \& Kalb. on bark of Sorbus aucuparia; $27\left[\mathrm{R}_{1}\right]$.

Pseudosagedia Chlorotica (Ach.) Hafellner \& Kalb. - on granite boulders; a16 $\left[\mathrm{R}_{1}\right]$.

Pseudoschismatomma Rufescens (Pers.) Ertz \& Tehler - on bark of Populus tremula; $9\left[\mathrm{R}_{1}\right]$.

Psilolechia Clavulifera (Nyl.) Coppins - on upturned roots; a4 $\left[\mathrm{R}_{1}\right]$.

Psilolechia lucida (Ach.) M. Choisy - on upturned roots and granite boulders; 19, 20, a4 [R]. Thalli contain atranorin, cf. chloratranorin and rhizocarpic acid.

PyCNORA SOROPHORA (Vain.) Hafellner - on wood of Pinus sylvestris; $27\left[\mathrm{R}_{1}\right]$.

RAMALINA FARINACEA (L.) Ach. - on bark of Alnus glutinosa, Picea abies, Pinus sylvestris, Populus tremula, Quercus robur, Sorbus aucuparia; 6, 9, 22, 27, 30, a15 [R].

RAMALINA FRAXINEA (L.) Ach. - on bark of Populus tremula, Sorbus aucuparia; 9, 16 [R].

RAMALINA SUBFARINACEA (Nyl. ex Cromb.) Nyl. - on bark of Picea abies and wood of Juniperus communis, on granite boulders; 15, 23, 27, 31, a11, a12 [R]. Coll. MB: 26.07.1868 (H s. n., 2 specimens).

Rhizocarpon Badioatrum (Flörke ex Spreng.) Th. Fr. - on granite boulder; $29\left[\mathrm{R}_{1}\right]$.

RHIZOCARPON DISPORUM (Nägeli ex Hepp) Müll. Arg. - on granite boulder; $15\left[\mathrm{R}_{1}\right]$.

RHIZOCARPON DISTINCTUM Th. Fr. - on granite boulders; 1, 11, 12, 16, 23, 26 [R].

RHIZOCARPON GEOGRAPHICUM (L.) DC. - on granite boulders; 1, 11, 12, 18, 23, 26, 31 [O].

RhizocARPon laVATUM (Fr.) Hazsl. - on granite boulders and brick; 7, 29 [R].

RHIZOCARPON LECANORINUM Anders - on granite boulders; 7, 18, 23, 26, 31 [R].

RHIZOCARPON MACROSPORUM Räsänen - on granite boulders and small pebbles; $8,16,18$, a14 [R].

RHIZOCARPON POLYCARPUM (Hepp) Th. Fr. - on granite boulder; $7\left[R_{1}\right]$.

RHIZOCARPON RICHARDII (Lamy ex Nyl.) Zahlbr. on granite boulders on seashore; $1,13,15$, 23, $31[R]$.

RHIZOCARPON RUBESCENS Th. Fr. - on granite boulder; $7\left[R_{1}\right]$. - New to WLR, previously known from ELR (Kuznetsova et al., 2007). 
RINODINA EXIGUA (Ach.) Gray - on wood and bones; 13, a12, a $17[R]$.

RINODINA GENNARII Bagl. - on granite boulder; $13\left[\mathrm{R}_{1}\right]$.

RINODINA PYRINA (Ach.) Arnold - on bark of Alnus glutinosa, Juniperus communis, on wood and bones; 16, a3, a7, a9, a12 [R].

RINODINA SEPTENTRIONALIS Malme - on bark of Rosa rugosa; $25\left[\mathrm{R}_{1}\right]$.

RiNODiNA SOPHODES (Ach.) A. Massal. - on bark of Acer platanoides, Alnus glutinosa, Juniperus communis, Sorbus aucuparia; 16, 25, a7 [R].

ROPALOSPORA VIRIDIS (Tønsberg) Tønsberg - on bark of Alnus glutinosa, Populus tremula, Sorbus aucuparia; 6, 9, 22, 27, 28, 30 [R].

Rufoplaca ARenaria (Pers.) Arup, Søchting \& Frödén - on concrete; a10 [ $\left.R_{1}\right]$.

SARCOGYNE REgULARIS Körb. - on concrete; a10 $\left[R_{1}\right]$.

+ SAREA Difformis (Fr.) Fr. - on resin of Picea abies; $28\left[\mathrm{R}_{1}\right]$.

+ SAREa Resinae (Fr.: Fr.) Kuntze - on resin of Picea abies; $21\left[\mathrm{R}_{1}\right]$.

SCHAERERIA FUSCOCINEREA (Nyl.) Clauzade \& $\mathrm{Cl}$. Roux - on granite boulders; 15, 18, 31 [R].

Scoliciosporum CHLOROCOCCUM (Graewe ex Stenh.) Vězda - on bark of Pinus sylvestris, Sorbus aucuparia, on wood of Picea abies and Pinus sylvestris; 9, 14, 16, 24, 27, 28 [R].

SCOLICIOSPORUM SAROTHAMNI (Vain.) Vězda - on bark of Alnus glutinosa, Betula pubescens, Juniperus communis, Picea abies, Pinus sylvestris, Populus tremula, Rosa rugosa, Syringa vulgaris, Sorbus aucuparia, on wood of Picea abies and Pinus sylvestris; 2, 4, 6, 7, 9, 14, 16, 19, 20, 22-25, 27, 28, 30, a7 [F].

SCOLICIOSPORUM UMBRINUm (Ach.) Arnold - on granite boulders; 1, 12, 15, 18, 31 [R].

\# Sphaerellothecium PRopinquellum (Nyl.) Cl. Roux \& Triebel - on apothecia of Lecanora carpinea on bark of Rosa rugosa; 25 [R $]$.

Steinia GEOPHANA (Nyl.) Stein - on primary soil on granite boulder; a17 $\left[\mathrm{R}_{1}\right]$.

+ Stenocybe PUllatula (Ach.) Stein - on bark of Alnus glutinosa; 6 [ $\left.\mathrm{R}_{1}\right]$.

STEREOCAULON ALPINUM Laurer - on soil, granite boulders, leather; $3,7,8,11,12,14,16$, 18, 26, a9, a14, a21 [O]. On soil in different localities (Liksakova \& Glazkova, 2018).

SteREOCAUlon CONDENSATUm Hoffm. - on sandy soil; 3, 5, 14, 32 [R].
Stereocaulon glareosum (L. I. Savicz) H. Magn. - on sandy soil; 3, 11, 14, 32, a12 [R]. Coll. EN: on sandy soil, 1851 (H 8003538).

STEREOCAULON INCRUSTATUM Flörke - on sandy soil; 3, 5, 32, a 14 [R].

Stereocaulon paschale (L.) Hoffm. - BL1 and BL2, on sandy soil (Lemberg, 1935).

Stereocaulon saxatile H. Magn. - on sandy soil, granite boulders, iron; 3, 7, 8 [R].

Stereocaulon taeniarum (H. Magn.) Kivistö - on sandy soil; $18\left[\mathrm{R}_{1}\right]$.

STEREOCAUlON TOMENTOSUM Fr. - on soil and granite boulders; 4, 12, 26, 29, a21 [R].

\# STigmidium fuscatae (Arnold) R. Sant. - on thalli of Acarospora fuscata on granite boulders; $7,16[R]$.

STRANGOSPORA MORIFORMIS (Ach.) Stein - on wood; 18 , a9 [R].

Strigula stigmatella (Ach.) R. C. Harris - on bark of Salix caprea; a20 [R $\mathrm{R}_{1}$. - New to WLR, previously known from ELR (Kuznetsova et al., 2012).

\# Talpapellis Beschiana (Diederich) Zhurb., U. Braun, Diederich \& Heuchert - on thallus of Cladonia uncialis subsp. biuncialis on sandy soil; $8\left[\mathrm{R}_{1}\right]$.

TEPHROMELA ATRA (Huds.) Hafellner - on granite boulders; 1, 13, 15, 18, 25, 31 [R].

THELENELla PeRTUSARIELla (Nyl.) Vain. - on bark of Acer platanoides; $25\left[\mathrm{R}_{1}\right]$.

THELOCARPON EPIBOLUM Nyl. - on wood of Pinus sylvestris; $27\left[\mathrm{R}_{1}\right]$.

TONINIOPSIS SUBINCOMPTA (Nyl.) Kistenich et al. - on bark and mosses on bark of Acer platanoides; 25 [ $\left.\mathrm{R}_{1}\right]$.

TRAPElia COARCTATA (Sm.) M. Choisy - on brick; $29\left[R_{1}\right]$.

TRAPElia Glebulosa (Sm.) J. R. Laundon - on brick; $29\left[R_{1}\right]$.

TRAPELIA PlaCodioIDEs Coppins \& P. James - on granite boulders and brick; 7, 29 [R].

TRAPELIOPSIS FleXUOSA (Fr.) Coppins \& P. James - on bark of Juniperus communis, Pinus sylvestris, on wood; $3,4,7,9,11-13,15$, $16,18,20,23,24,31$, a6 [F].

TRAPELIOPSIS GRANULOSA (Hoffm.) Lumbsch - on soil, wood, rubber; 3-5, 7-9, 11, 14, 18, 26, 29, $32[\mathrm{O}]$.

\# Tremella lichenicola Diederich - on thallus of Violella fucata on lignum of Picea abies; $21\left[\mathrm{R}_{1}\right]$.

TUCKERMANNOPSIS CHLOROPHYLLA (Willd. ex Humb.) Hale - on bark of Alnus glutinosa, Juniperus 
communis, Picea abies, Pinus sylvestris, on wood; 4, 18-21, 23, 26-28, 30 [O]. Coll. NB: on bark of tree, 1993 (Alexeeva, 2005).

UMBILICARIA DEUSTA (L.) Baumg. - on granite boulders; $7,15,16,18,23,31$, a1 [R].

UMBILICARIA POLYPHYLLA (L.) Baumg. - on granite boulders; $7,12,15,16,18,23,31$, a1 [O].

UMBILICARIA PROBOSCIDEA (L.) Schrad. - on granite boulders; $18\left[\mathrm{R}_{1}\right]$. Red Data Book of LR (Red..., 2018).

UMBILICARIA TORREFACTA (Lightf.) Schrad. - on granite boulders; 7, 11, 15, 16, 18, 23 [R].

USNEA DASOPOGA (Ach.) Nyl. - on bark of Alnus glutinosa; $30\left[\mathrm{R}_{1}\right]$.

UsNeA HIRTA (L.) F. H. Wigg. - on bark of Picea abies, Pinus sylvestris, on wood of Pinus sylvestris; 18-20, 24, 27 [R].

USNEA SUBFLORIDANA Stirt. - on bark of Alnus glutinosa, Sorbus aucuparia; 22, 30 [R].

VERRUCARIA DOLOSA Hepp - on granite boulder; a16 $\left[R_{1}\right]$.

VERRUCARIA FUSCONIGRESENS Nyl. - on seashore granite boulder; $31\left[R_{1}\right]$.

VERRUCARIA MURALIS Ach. - on brick, concrete; 29, a10 [R].

VERRUCARIA XYLOXENA Norman - on sandy soil; $29\left[R_{1}\right]$.

Violella fucata (Stirt.) T. Sprib. - on bark of Picea abies, Pinus sylvestris, on wood of Picea abies; 2, 21, 27 [R].

VUlPICIDA PINASTRI (Scop.) J.-E. Mattsson \& M. J. Lai - on bark of Alnus glutinosa, Juniperus communis, Pinus sylvestris, Sorbus aucuparia, on wood; $2,4,6,7,9,11,14,18,20$, 21, 23, 24, 27, 28, 30 [F]. Coll. NB: on bark of tree, 1993 (Alexeeva, 2005).

XanthocarPia CRenulatella (Nyl.) Frödén, Arup \& Søchting - on concrete; a10 $\left[\mathrm{R}_{1}\right]$.

XANTHOPARMElia CONSPERSA (Ehrh. ex Ach.) Hale on bark of Juniperus communis, on granite boulders; 1, 7, 11-13, 15-18, 23, 25, 26, $31[\mathrm{~F}]$.

XANTHOPARMElia LOXOdes (Nyl.) O. Blanco et al. on granite boulders; 12,23 , a12 [R]. Thalli contain perlatolic, glomellic and glomelliferic acids. Red Data Book of LR (Red..., 2018).

XANTHOPARMELIA PULLA (Ach.) O. Blanco et al. - on granite boulders; $1,10,12,13,15,16,18$, 23, $31[\mathrm{O}]$. Thalli contain perlatolic, glomellic and glomelliferic acids. Red Data Book of LR (Red..., 2018).

Xanthoparmelia stenophylla (Ach.) Ahti \& D. Hawksw. - on granite boulders; 1, 7, 11, 12, $15,16,18,23,25,31$, a4 [O].
Xanthoria PARietina (L.) Th. Fr. - on bark of Acer platanoides, Alnus glutinosa, Juniperus communis, Malus domestica, Populus tremula, Syringa vulgaris, Sorbus aucuparia, on wood, granite boulders, concrete, bones; $1,9,10,13,15-17,22,23,25,31$, a3, a7, a9, a10, a12, a21 [O]. Coll. NB: on bark of deciduous tree, 1993 (Alexeeva, 2005).

\# XANTHORIICOLA PHYSCIAE (Kalchbr.) D. Hawksw. on apothecia of Xanthoria parietina on bark of Acer platanoides and Populus tremula; 9, $25[R]$.

\# Xenonectriella leptaleae (J. Steiner) Rossman \& Lowen - on apothecia of Physcia aipolia on bark of Acer platanoides; $25\left[\mathrm{R}_{1}\right]$.

XYLOGRAPHA OPEGRAPHELla Nyl. ex Rothr. - on wood; 1, 4, 11, 13, 15, 31 [R]. Thalli contain norstictic and connorstictic acids, traces of stictic acid.

XYlographa PARAllela (Ach.: Fr.) Fr. - on wood; 4, 23, a9 [R].

XYLOPSORA FRIESII (Ach.) Bendiksby \& Timdal on bark of Picea abies, Pinus sylvestris; 20, $21,28[R]$.

\section{DISCUSSION}

The documented lichen flora of Moshchny Island is moderately rich, with a total of 349 species (313 lichens, 30 lichenicolous fungi and 6 non-lichenized saprobic fungi), practically all of which have been recorded recently (348 species). These numbers are high, taking into account the relatively small size of the island, the lack of rocky outcrops, and the long history of anthropogenic transformation of the ecosystems. The detected lichen diversity of the island is comparable to the diversity of neighbouring Tuters Island (335 species; Stepanchikova et al., 2017; Himelbrant et al., 2019) and the whole Berezovye Archipelago (356 species; Stepanchikova et al., 2011) in Gulf of Vyborg, as well as the rocky Valaam Archipelago (363 species; Stepanchikova \& Himelbrant, 2004) in the northern part of Lake Ladoga. On the other hand, the lichen diversity of the relatively small non-rocky Konevets Island in the western part of Lake Ladoga is much higher (438 species; Himelbrant et al., 2018, 2019).

Two species of lichenicolous fungi (Endococcus exerrans and Lichenopeltella coppinsii) reported in the present paper are new for Russia, three li- 
chens (Flavoplaca limonia, Lecidea haerjedalica, and Myriospora myochroa) and two lichenicolous fungi (Cercidospora stenotropae and Erythricium aurantiacum) are new for European Russia, two lichens (Flavoplaca oasis and Myriolecis zosterae) and two lichenicolous fungi (Intralichen christiansenii and Nesolechia fusca) are new for the North-Western European Russia, two lichens (Calogaya pusilla and Lecidea auriculata subsp. auriculata) and one lichenicolous fungus (Arthrorhaphis aeruginosa) are new for LR, while five lichens (Flavoplaca dichroa, Myriolecis perpruinosa, Polycauliona phlogina, Rhizocarpon rubescens and Strigula stigmatella) and one lichenicolous fungus (Nectriopsis lecanodes) are reported for the first time for WLR.

The majority of the species recorded in Moshchny Island in 2017 are rare [R] (277 species, $79.4 \%$ of the modern lichen flora), 124 (35.5\%) of which were recorded only once $\left[\mathrm{R}_{1}\right]$, while 56 species $(16.1 \%)$ were occasional [O], 14 species $(4.0 \%)$ frequent $[\mathrm{F}]$, one species $(0.3 \%)$ common [C] (Hypogymnia physodes), and none is very common [VC].

Of 19 lichen species known for Moshchny Island on the base of collections or records made in 19 th and 20th centuries, Stereocaulon paschale is the only one which was not found during our investigations. The species was recorded by Lemberg (1935) from two recently reinvestigated localities (BL1 and BL2), but we found no confirming specimens in revised lichen herbaria. However, we can assume that this very common and widespread species could occur in Moshchny Island, because suitable habitats are still present there. Unfortunately, the lack of data on historical lichen diversity in the island renders impossible any floristic comparison and estimation of nature transformation.

Most of the lichens recorded in Moshchny in 2017 are corticolous (143 species, $41.0 \%$ of the lichen flora), saxicolous (117 species, 33.5\%) or lignicolous (105 species, 30.1\%). The lichens on soil and plant debris are quite abundant in different parts of the island, but their diversity is not very high (65 species, 18.6\%). Some species grow also on old bones ( 21 species), iron (10 species), mosses (8 species), old leather (2 species), rubber (2 species) and resin of conifers (2 species). The richest phorophytes are Pinus sylvestris (62 species, $17.7 \%$ of the lichen flora),
Alnus glutinosa (61 species, 17.5\%), Sorbus aucuparia (48 species, 13.8\%), Populus tremula (41 species, $11.7 \%$ ), Picea abies (40 species, $11.5 \%$ ), Juniperus communis (38 species, 10.9\%). The other phorophytes include Acer platanoides (25 species, 7.2\%), Quercus robur (19 species, $5.4 \%$ ), Betula pubescens (13 species, 3.7\%), Malus domestica, Rosa rugosa and Syringa vulgaris (6 species, $1.7 \%$ each), and Salix caprea (3 species, $0.9 \%$ ). The lichens on granite (96 species, $27.4 \%$ ) are much more diverse than on concrete (34 species, 9.7\%). A rather high percent of lichenicolous species (30 species, 8.6\% of species diversity) indicates, in our opinion, that the lichen diversity of the island nowadays is comparatively well studied.

Most of the lichen species were recorded in pine forests and open pine stands, which are widespread in the island (altogether 219 species, $62.8 \%$ of the lichen flora). Also different anthropogenic communities were very rich, represented mainly by wastelands, old buildings, piers, and old cemetery (153 species, 43.8\%). Natural seashore communities were inhabited by 152 species (43.6\%), most of them being saxicolous. Also quite many lichens were found in black alder stands (75 species, $21.5 \%$ ), or in aspen (55 species, $15.8 \%$ ) and spruce forests (54 species, $15.5 \%$ ), while few were recorded in mixed forests (19 species, 5.4\%).

Most interesting and valuable habitats in Moshchny Island occur on the seashore. Contrary to inland forest communities, seashores bear the majority of the lichen diversity, including some rare saxicolous, terricolous and lignicolous species. The seashore habitats contain very specific saxicolous lichen communities with evident marine features (such as Amandinea cacuminum, A. coniops, Flavoplaca marina, Hydropunctaria maura, Lecanora helicopis, Myriolecis salina s. 1., Rhizocarpon richardii, and Xanthoparmelia pulla). Of special interest are also the sand dunes and sandy seashores overgrown by pine stands, in most cases with abundant and diverse lichens on soil. In such communities, for example, the red-listed (in LR) Cladonia scabriuscula and C. macrophylla were recorded. Moreover, a rather important part of the lichen diversity of Moshchny is confined to old anthropogenic wastelands and buildings.

The forests of Moshchny are secondary, thus not old-growth, and lichens in them are mostly 
represented by common, widespread species. We recorded only one habitat specialist of oldgrowth biologically valuable forests (Andersson et al., 2009) - Acrocordia cavata, and three indicator species - Arthonia spadicea, Calicium tigillare, and Chaenothecopsis nigra. All of them are rare, but widely distributed in LR. These species were not found together, but scattered in different localities and habitats, and their single records are not diagnostic for biologically valuable forests. However, a small patch of relatively untouched black alder forest with aspen in a wet brook valley was observed in the central part of Moshchny N of Bol'shoe Lake (sample area 22) but probably this is the only exception. There we found three red-listed lichen species (Acrocordia cavata, Arthonia spadicea, and Phaeophyscia endophoenicea), two of which are species associated with old-growth forests. Tentatively this forest community could be estimated as biologically valuable. The known historical collections or literature records do not include any data on indicator species and habitat specialists from Moshchny Island.

Altogether 11 species nowadays known from Moshchny Island are included in the Red Data Book of Leningrad Region (Red..., 2018): Acrocordia cavata, Arthonia spadicea, Calicium tigillare, Cetrariella commixta, Cladonia macrophylla, C. scabriuscula, Melanelia stygia, Phaeophyscia endophoenicea, Umbilicaria proboscidea, Xanthoparmelia loxodes, and X. pulla. Of these, two corticolous species, Acrocordia cavata and Arthonia spadicea, in LR are restricted to relatively undisturbed, mostly deciduous forests (Andersson et al., 2009). They are quite rare in LR and distributed mostly in the south-western part of the region, the second one being most abundant in natural black alder stands near the sea. One more epiphytic lichen, Phaeophyscia endophoenicea, is known from a few localities in southern part of the LR and SPb, where it grows in deciduous forests. The lignicolous Calicium tigillare was previously reported from different localities in LR but is nowadays extremely rare and only known from the islands in the Gulf of Finland. The saxicolous species mentioned above occur almost exclusively in the western part of LR. Cetrariella commixta, Melanelia stygia, and Umbilicaria proboscidea are confined to rocky outcrops and large seashore boulders. Xanthoparmelia loxodes and X. pulla are mainly seashore species. Of the two Cladonia species occurring on soil, C. scabriuscula is known in LR only from the islands in the Gulf of Finland and C. macrophylla is confined to open pine stands, rocky outcrops and sometimes wastelands, also mostly in the western part of the region. All the protected lichen species were recorded in Moshchny Island rarely $[R]$ or once $\left[R_{1}\right]$ only.

To conclude, the Moshchny Island nowadays bears a moderately rich lichen biota with the most valuable lichen habitats confined to the seashore. In our opinion this island is worth being incorporated in the nature reserve 'Eastern Gulf of Finland' which already includes several neighbouring islands.

\section{ACKNOWLEDGEMENTS}

We are grateful to the Complex Expedition 'Gogland' of the Russian Geographical Society (RGS) for organizing the fieldwork, and we thank very much all participants of 2017 trip to Moshchny Island for support and co-operation. We would like to thank Ludmila Konoreva and Sergey Chesnokov (Komarov Botanical Institute of Russian Academy of Sciences, St. Petersburg) for revision of Micarea specimens, and Wolfgang von Brackel (Röttenbach, Germany) for identification of Endococcus exerrans and Lichenopeltella coppinsi. We are grateful to Mikhail P. Zhurbenko (Komarov Botanical Institute, Russia) for valuable comments on distribution of lichenicolous fungi in Russia and Ivan V. Frolov (Botanical Garden of the Urals Branch of Russian Academy of Sciences, Yekaterinburg) for comments on distribution of Caloplaca s. 1. group in European Russia. We appreciate the help of colleagues in the Botanical Museum, University of Helsinki during our work in $\mathrm{H}$ herbarium. The authors are grateful to an anonymous reviewer for valuable corrections. The study was carried out within the framework of the institutional research project 'Flora of lichens and bryophytes of Russia and phytogeographically important regions' (no. AAAA-A19-119020690077-4) of the Komarov Botanical Institute of the Russian Academy of Sciences.

\section{REFERENCES}

Alexeeva, N. 2005. Lichens from islands in the Russian part of the Gulf of Finland. Folia Cryptogamica Estonica 41: 5-12. 
Andersson, L., Alexeeva, N. M. \& Kuznetsova, E. S. (eds.). 2009. Survey of biologically valuable forests in North-Western European Russia. Vol. 2. Identification manual of species to be used during survey at stand level. St. Petersburg. 258 pp. (In Russian).

Arup, U. 2009. The Caloplaca holocarpa group in the Nordic countries, except Iceland. Lichenologist 41(2): 111-130. https://doi.org/10.1017/ S0024282909008135

Arup, U. 2011. Contributions to the knowledge of Caloplaca in the Nordic countries. Graphis Scripta 23: 10-20.

Calatayud, V., Navarro-Rosinés, P. \& Hafellner, J. 2013. Contributions to a revision of Cercidospora (Dothideales), 2: Species on Lecanora s. 1., Rhizoplaca and Squamarina. Mycosphere 4: 539-557. https://doi.org/10.5943/mycosphere/4/3/8

Diederich, P. 2003. New species and new records of American lichenicolous fungi. Herzogia 16: 41-90.

Diederich, P. Lawrey, J. D. \& Ertz, D. 2018. The 2018 classification and checklist of lichenicolous fungi, with 2000 non-lichenized, obligately lichenicolous taxa. PLoS ONE 121: 340-426. https://doi. org/10.1639/0007-2745-121.3.340

Diederich, P., Schultheis, B. \& Blackwell, M. 2003: Marchandiobasidium aurantiacum gen. sp. nov., the teleomorph of Marchandiomyces aurantiacus (Basidiomycota, Ceratobasidiales). Mycological Research 107: 523-527. https://doi.org/10.1017/ S0953756203007639

Earland-Bennett, P. M. \& Hawksworth, D. L. 1999. Lichenopeltella coppinsii, a new species on Verrucaria muralis from the British Isles. Lichenologist 31(6): 575-578. https://doi.org/10.1006/ lich.1999.0218

Fadeeva, M. A., Golubkova, N. S., Vitikainen, O. \& Ahti, T. 2007. Conspectus of lichens and lichenicolous fungi of the Republic of Karelia. Petrozavodsk. 194 pp. (In Russian, English summary).

Hafellner, J. \& Türk, R. 2016. Die lichenisierten Pilze Österreichs - eine neue Checkliste der bisher nachgewiesenen Taxa mit Angaben zur Verbreitung und Substratökologie. Stapfia 104(1): 1-216.

Hawksworth, D. L. 1983. A key to the lichen-forming, parasitic, parasymbiotic and saprophytic fungi occurring on lichens in the British Isles. Lichenologist 15(1): 1-44. https://doi.org/10.1017/ S0024282983000031

Himelbrant, D. E., Stepanchikova, I. S., Kuznetsova, E. S., Motiejūnaitė, J. \& Konoreva, L. A. 2018. Konevets Island (Leningrad Region, Russia) - a historical refuge of lichen diversity in Lake Ladoga. Folia Cryptogamica Estonica 55: 51-78. https:// doi.org/10.12697/fce.2018.55.07

Himelbrant, D. E., Stepanchikova, I. S., Motiejūnaitè, J., Kuznetsova, E. S., Tagirdzhanova, G. \& Frolov, I. V. 2019. New records of lichens and allied fungi from the Leningrad Region, Russia. X. Folia Cryptogamica Estonica 56 (in rev.).
Himelbrant, D. E., Stepanchikova, I. S., Motiejūnaite, J., Vondrak, J., Tagirdzhanova, G. M., Gagarina, L. V. \& Kuznetsova, E. S. 2015. New records of lichens and allied fungi from the Leningrad Region, Russia. VI. Folia Cryptogamica Estonica 52: 21-28. https:/ / doi.org/ 10.12697 / fce.2015.52.03

Gaya, E. 2009. Taxonomical revision of the Caloplaca saxicola Group (Teloschistaceae, lichen-forming Ascomycota). Bibliotheca Lichenologica 101: $1-191$.

Knudsen, K., Kocourková, J. \& Schiefelbein, U. 2017. New reports of Myriospora (Acarosporaceae) from Europe. Mycotaxon 132: 857-865. https://doi. org/ 10.5248/132.857

Kotiranta, H., Uotila, P., Sulkava, S. \& Peltonen, S.-L. (eds). 1998. Red Data Book of East Fennoscandia. Helsinki. 351 pp.

Kukwa, M. 2011. The lichen genus Ochrolechia in Europe. Gdańsk. 309 pp.

Kuznetsova, E., Ahti, T. \& Himelbrant, D. 2007. Lichens and allied fungi of the Eastern Leningrad Region. Norrlinia 16: 1-62.

Kuznetsova, E. S., Kataeva, O. A., Himelbrant, D. E. \& Motiejūnaitè, J. 2016. Lichens and allied fungi of the Ragusha River Protected Area (Leningrad Region, Russia). Folia Cryptogamica Estonica 53: 71-80. https:/ / doi.org/10.12697/fce.2016.53.09

Kuznetsova, E. S., Motiejūnaitè, J., Stepanchikova, I. S., Himelbrant, D. E. \& Czarnota, P. 2012. New records of lichens and allied fungi from the Leningrad Region, Russia. III. Folia Cryptogamica Estonica 49: 31-37.

Lawrey, J. D. \& Diederich, P. 2018. Lichenicolous fungi - worldwide checklist, including isolated cultures and sequences available. URL: http:// www.lichenicolous.net (25 March 2019).

Lemberg, B. 1935. Über die Vegetation der Flugsandgebiete an den Küsten Finnlands. III Die einzelnen Flugsangebiete. Acta Botanica Fennica 14: 1-75.

Liksakova, N. S. \& Glazkova, E. A. 2018. Vegetation and conservation value of plant communities and biotopes of Lavansaari Island (Gulf of Finland). Botanicheskii Zhurnal 103(10): 1304-1335. https:/ / doi.org/10.7868/S0006813618100083

Magnusson, A. H. 1948. New or otherwise interesting swedish lichens XIII. Botaniska Notiser 4: 401-412.

Makryi, T. V. 2005. To the Lichen Flora of the Stanovoye Nagorye Uplands (Baikalian Siberia). II. The additions to the flora of epilithic lichens of the Kodar Range. Turczaninowia 8(3): 60-66. (In Russian, English summary).

Motiejūnaitè, J., 2017. Supplemented checklist of lichens and allied fungi of Lithuania. Botanica Lithuanica 23(2): 89-106. https://doi.org/10.1515/ botlit-2017-0011

Motiejūnaitè, J., Chesnokov, S. V., Czarnota, P., Gagarina, L. V., Frolov, I., Himelbrant, D., Konoreva, L. A., Kubiak, D., Kukwa, M., Moisejevs, R., Stepanchikova, I., Suija, A., Tagirdzhanova, G., 
Thell, A. \& Tsurykau, A. 2016. Ninety-one species of lichens and allied fungi new for Latvia with a list of additional records from Kurzeme. Herzogia 29(1): 143-163. https://doi.org/10.13158/ heia.29.1.2016.143

Muchnik, E. A. 2016. Additions to lichen biota of Moscow Region. Proceedings of Petrozavodsk State University 8(161): 52-57. (In Russian, English abstract).

Nordin, A., Moberg, R., Tønsberg, T., Vitikainen, O., Dalsätt, Å., Myrdal, M., Snitting, D. \& Ekman, S. 2011. Santesson's Checklist of Fennoscandian Lichen-forming and Lichenicolous Fungi. Ver. April 29, 2011 http://130.238.83.220/santesson/ home.php (25 March 2019).

Orange, A., James, P. W. \& White, F. J. 2001. Microchemical methods for the identification of lichens. London. $101 \mathrm{pp}$.

Øvstedal, D. G., Tønsberg, T. \& Elvebakk, A. 2009. Lichen flora of Svalbard. Sommerfeltia 33: 1-393. https://doi.org/10.2478/v10208-011-0013-5

Piispa, H. 2013. Lavansaari. Itäisen Suomenlahden villit vuodet. Kirjokansi 32: 1-217.

Randlane, T., Saag, A. \& Suija, A. 2018. Lichenized, lichenicolous and allied fungi of Estonia. Ver. December 31, 2018 - http:/ /esamba.bo.bg.ut.ee/ checklist/est/home.php (25 March 2019).

Red Data Book of the Lenindrad Region. St. Petersburg. 848 pp. (In Russian).

Santesson, R. \& Tønsberg, T. 1994. Arthrorhaphis aeruginosa and $A$. olivacea, two new lichenicolous fungi. Lichenologist 26(3): 295-299. https:/ / doi. org/10.1006/lich.1994.1021

Schiefelbein, U., Brackel, W. v., Cezanne, R., Czarnota, P., Eckstein, J., Eichler, M., Kison, H.-U., Ungethüm, K. \& Stordeur, R. 2017. Trimmatostroma arctoparmeliae sp. nov. and noteworthy records of lichenized, lichenicolous and allied fungi from the Harz Mountains and surrounding regions. Herzogia 30: 80-102. https://doi.org/10.13158/ heia.30.1.2017.80

Schiefelbein, U., Dolnik, C., de Bruyn, U., Schultz, M., Thiemann, R., Stordeur, R., van den Boom, P. P. G., Litterski, B. \& Sipman, H. J. M. 2014. Interesting records of lichenized, lichenicolous and saprophytic fungi from Northern Germany. Herzogia 27: 237-256. https://doi.org/10.13158/ heia.27.2.2014.237

Schiefelbein, U., Dolnik, C. \& Westberg, M. 2015. The lichen genus Myriospora in the Baltic coastal zone of Germany. Graphis Scripta 27(1-2): 27-31.

Śliwa, L. 2007. A revision of the Lecanora dispersa complex in North America. Polish Botanical Journal 52(1): 1-70.

Smith, C. W., Aptroot, A., Coppins, B. J., Fletcher, A., Gilbert, O. L., James, P. W. \& Wolseley, P. A. (eds). 2009. The lichens of Great Britain and Ireland. The British Lichen Society, London. 1046 pp.

Stepanchikova, I. S., Andreev, M. P., Himelbrant, D. E., Motiejūnaitė, J., Schiefelbein, U., Konoreva, L.
A. \& Ahti, T. 2017. The lichens of Bolshoy Tuters Island (Tytärsaari), Leningrad Region, Russia. Folia Cryptogamica Estonica 54: 95-116. https:/ / doi.org/10.12697/fce.2017.54.14

Stepanchikova, I. S. \& Himelbrant, D. E. 2004. The history of research and contemporary knowledge on the lichens of Valaam Archipelago (Karelia ladogensis). Proceedings of VIII Youth Conference of botanists in St. Petersburg 17-21 may 2004. St. Petersburg. P. 87. (In Russian).

Stepanchikova, I. S., Schiefelbein, U., Alexeeva, N. M., Ahti, T., Kukwa, M., Himelbrant, D. E. \& Pykälä, J. 2011. Additions to the lichen biota of Berezovye Islands, Leningrad Region, Russia. Folia Cryptogamica Estonica 48: 95-106.

Triebel, D., Rambold, G. \& Elix, J. A. 1995. A conspectus of the genus Phacopsis (Lecanorales). Bryologist 98: 71-83. https://doi.org/10.2307/3243643

Tsurykau, A. \& Korchikov, E. S. 2017. Lichenicolous fungi from the Samara Region, southern part of European Russia. Folia Cryptogamica Estonica 54: 1-8. https:/ / doi.org/ 10.12697/fce.2017.54.01

Urbanavichus, G. P., Ahti, T. \& Urbanavichene, I. N. 2008. Catalogue of lichens and allied fungi of Murmansk Region, Russia. Norrlinia 17: 1-80.

Vondrák, J., Frolov, I., Davydov, E. A., Urbanavichene, I., Chesnokov, S., Zhdanov, I., Muchnik, E., Konoreva, L., Himelbrant, D. \& Tchabanenko, S. 2016. The extensive geographical range of several species of Teloschistaceae: evidence from Russia. Lichenologist 48(3): 171-189. https://doi. org/10.1017/S0024282916000116

Vondrák, J., Redchenko, O., Himelbrant, D., Stepanchikova, I. \& Kuznetsova, E. 2010. Some sterile Caloplaca crusts identified by molecular data from the Leningrad region (Russia). Folia Cryptogamica Estonica 47: 97-99.

Vondrák, J., Řiha, P., Arup, U. \& Søchting, U. 2009. The taxonomy of the Caloplaca citrina group (Teloschistaceae) in the Black Sea region; with contributions to the cryptic species concept in lichenology. Lichenologist 41(6): 571-604. https:/ / doi.org/10.1017/S0024282909008317

Westberg, M., Crewe, A. T., Purvis, O. W. \& Wedin, M. 2010. Silobia, a new genus for the Acarospora smaragdula complex (Ascomycota, Acarosporales) and a revision of the group in Sweden. $\mathrm{Li}$ chenologist 43(1): 7-25. https://doi.org/10.1017/ S0024282910000617

Wirth, V., Hauck, M. \& Schultz, M. 2013. Die Flechten Deutschlands. Band 1. Eugen Ulmer, Stuttgart. $672 \mathrm{pp}$.

Zhdanov, I. 2013. Additions to the lichen flora of Central Siberian Biosphere Reserve (Krasnoyarsk Territory). Novitates Systematicae Plantarum Non Vascularium 47: 200-214. (In Russian, English abstract).

Zhurbenko, M. P. 2008. Lichenicolous fungi from Russia, mainly from its Arctic. II. Mycologia Balcanica 5(1-2): 13-22. 
Zhurbenko, M. P. 2009. Lichenicolous fungi and lichens from the Holarctic. Part II. Opuscula Philolichenum 7: 121-186.

Zhurbenko, M. P. \& Kobzeva, A. A. 2014. Lichenicolous fungi from Northwest Caucasus, Russia. Herzogia 27: 377-396. https://doi.org/10.13158/ heia.27.2.2014.377
Zhurbenko, M. P. \& Kobzeva, A. A. 2016. Further contributions to the knowledge of lichenicolous fungi and lichenicolous lichens of the Northwest Caucasus, Russia. Opuscula Philolichenum 15: 37-55.

Appendix 1. List of sampling locations in Leningrad Region, Kingisepp District, Moshchny Island (Lavansaari)

\begin{tabular}{ll}
\hline No $\quad$ Description, geographical coordinates, biotope & Date
\end{tabular}

Standard sample areas:

1 Promezhutochny Peninsula (Suisaari), S part, central part of Cape Linejny (Rivinnenä), 5959’00.9”N, 2753’27.8”E, 12.08 .2017 open sandy seashore with granite boulders

2 Promezhutochny Peninsula (Suisaari), central part, W of Cape Shumny (Elinäisennenä), 5959’46.0”N, 2753’32.1”E, 12.08.2017 young pine stand with sparse spruces with Vaccinium vitis-idaea L., Avenella flexuosa (L.) Drejer, and Pleurozium schreberi (Brid.) Mitt.

3 Promezhutochny Peninsula (Suisaari), central part, E of Hiekkataipale Isthmus, 6000'09.7”N, 2753’02.6”E, lichen 12.08 .2017 community on sandy seashore

4 Promezhutochny Peninsula (Suisaari), E shore, N of Cape Ostry (Tukkinaisennenä), 6000’17.4”N, 2753'43.1”E, 12.08 .2017 seashore young open pine stand with lichens, mosses, and Avenella flexuosa

5 Promezhutochny Peninsula (Suisaari), E of Hiekkataipale Isthmus, 6000’21.0”N, 2752’42.0”E, moss-lichen com- 12.08 .2017 munity on sand

6 Central part, NE shore of Bol'shoe Lake (Suurjärvi), 5959’55.8”N, 2749’45.7”E, young (ca. 30 year old) black alder 13.08 .2017 stand with older birch trees and sparse rowan, with Viola sp. and Oxalis acetosella L.

7 E of Bol'shoe Lake (Suurjärvi), 5959'31.4”N, 2750’22.6”'E, old construction (boulder ridge) in young (30-35 year 13.08.2017 old) pine stand with mosses, Avenella flexuosa and Melampyrum sp. (on previously burnt area)

8 SE shore (Heposelän rivit), 59 59'11.0”'N, 2750'23.1”'E, open pine stand on sandy dune with lichens and Avenella flexuosa 13.08 .2017

9 S part, S of Bol'shoe Lake (Suurjärvi), E of Maloe Lake (Pienjärvi), 5959’09.4”N, 2749’52.8”E, relatively young (50-60 13.08.2017 year old) aspen forest with Calamagrostis sp., Vaccinium vitis-idaea, and Maianthemum bifolium (L.) F. W. Schmidt

10 S shore, Cape Opasny (Kalmanniemi), 5958'53.8”N, 2749'38.9”'E, open sandy seashore with granite boulders

13.08.2017

11 S shore, Cape Kiipunaisenniemi, 5958'57.9”N, 2749’23.5”E, open pebble seashore

13.08.2017

12 Promezhutochny Peninsula (Suisaari), N part, Cape Pology (Keinäsnenä), 6000`46.4”N, 2753’08.0”E, glade in young 14.08 .2017 open pine stand with rowan shrubs, Avenella flexuosa, and lichens

13 Promezhutochny Peninsula (Suisaari), NNW part, N end of Vjunok Island (Mousoori), 6001'09.6”N, 2752'37.3”E, 14.08.2017 open seashore with granite boulders and pebbles

14 Central part of Hiekkataipale Isthmus, 6000’13.7”N, 2752’24.6”E, open pine stand with lichens on sand

15 N end, Kirkorvi Spit (Kierkoori), Cape Severny (Puokkiniemi), 6002’12.4”N, 2749'35.6”E, open sand-pebble seashore 15.08 .2017 with granite boulders

$16 \mathrm{~N}$ end, central part of Kirkorvi Spit (Kierkoori), 6002'02.1”N, 27 49'37.6”'E, open young pine stand with sparse 15.08 .2017 Juniperus communis, with lichens, Avenella flexuosa, granite boulders, and plots of open sand

17 N end, Lankoori Spit, $60^{\circ} 01^{\prime} 44.8^{\prime \prime} \mathrm{N}, 27^{\circ} 48^{\prime} 44.8^{\prime \prime} \mathrm{E}$, granite pier and digue

18 SW part, NE of Päkinlahti Bay, 59 59'29.8”N, 2748’30.3”E, lichen glade surrounded by pine forest

19 SW part, S shore of swamp NE of Cape Uglovoj (Ristiniemi), 5959'51.1”N, 2748’28.6”E, young (45-60 year old) 16.08 .2017 spruce forest with single birches, with mosses, near the swamp

20 SW part, W of Bol'shoe Lake (Suurjärvi), 59 59'41.5” N, $27^{\circ} 48^{\prime} 50.6^{\prime \prime}$, old (up to 140 year old) pine forest with 16.08 .2017 Melampyrum sp., green mosses, and Avenella flexuosa, with granite boulders

21 SW part, NW of Bol'shoe Lake (Suurjärvi), 59 $59^{\prime} 58.4{ }^{\prime} \mathrm{N}, 27^{\circ} 49^{\prime} 01.9^{\prime \prime}$ E, relatively old spruce forest (spruces up to 16.08 .2017 140 year old) with pines, with Vaccinium myrtillus L., Oxalis acetosella, Hylocomium splendens (Hedw.) Schimp., and Pleurozium schreberi

22 Central part, $\mathrm{N}$ of Bol'shoe Lake (Suurjärvi), 6000'09.8”N, $27^{\circ} 49^{\prime} 34.3^{\prime \prime} \mathrm{E}$, black alder stand with aspen in wet valley 16.08 .2017 of a brook, with Oxalis acetosella and Maianthemum bifolium 
23 NE part, W part of Cape Shilo (Eistoori, Torniluoto), 6001'21.0”N, 2747’49.2”E, open pine woodland with Juniperus 17.08 .2017 communis and Rosa rugosa, with lichens, mosses, Avenella flexuosa, and Hypericum sp., with granite boulders

24 NE part, S part of Cape Shilo (Eistoori), 6001’06.4”N, 27 48’18.3”E, old-growth (up to ca. 180 year old) pine forest 17.08 .2017 with green mosses, Melampyrum sp., and Avenella flexuosa

25 N part, former village Lounatkylä, $60^{\circ} 01^{\prime} 10.3^{\prime \prime} \mathrm{N}, 27^{\circ} 48^{\prime} 55.2^{\prime \prime}$, old foundation with maples, lilac, apple trees, and 17.08 .2017 Rosa rugosa shrubs

26 N part, former village Pohjakylä, near the lighthouse, 60 01’23.6” N, 27 49’31.2”E, anthropogenic wasteland with 17.08 .2017 Avenella flexuosa, lichens, and mosses, with old stony foundation

27 N shore, E of Cape Mustaniemi, 6001'16.2”N , 2750’39.8”E, young (40-45 year old) pine forest with sparse rowan 18.08 .2017 undergrowth, with mosses, and Vaccinium myrtillus

28 Central part, Vehnämaa, 600'44.7”N, 2750'12.0”'E, spruce forest with pines, with green mosses, Maianthemum 18.08 .2017 bifolium, Vaccinium myrtillus, and Oxalis acetosella. Some trees are old (up to ca. 150-170 year old), but the traces of former cuttings present

29 Central part, W of Okol'naya Bay (Pohjois-Kappalahti), 6000’29.3”N, 2751’02.6”E, anthropogenic wasteland with 18.08 .2017 Calluna vulgaris (L.) Hull, lichens, and mosses in former sandpit with brick, slate, granite boulders, and pebble

30 Central part, W of Hiekkataipale Isthmus, $60^{\circ} 00^{\prime} 08.4^{\prime} \mathrm{N}, 27^{\circ} 50^{\prime} 43.1^{\prime} \mathrm{E}$, black alder forest with ferns, surrounded by 20.08 .2017 spruce forest

31 S part, W shore of Zashchitnaya Bay (Lounat-Kappalahti), near Cape Liiskuniemi, 5959’28.1”N, 2751'17.5”E, open 20.08.2017 sandy shore with granite boulders

32 W part of Hiekkataipale Isthmus, Vanhankirkonmäki, $60^{\circ} 00^{\prime} 11.5^{\prime \prime} \mathrm{N}, 27^{\circ} 51^{\prime} 50.3^{\prime \prime} \mathrm{E}$, moss-lichen glade surrounded by 20.08 .2017 open pine stand on sandy dune

Additional plots:

a1 Promezhutochny Peninsula (Suisaari), S part, N part of Cape Linejny (Rivinnenä), 5959'04.6”N, 2753’28.0”E, open 12.08 .2017 sandy shore with granite boulders

a2 Promezhutochny Peninsula (Suisaari), central part, W of Cape Shumny (Elinäisennenä), 5959'50.3”N, 2753’21.6”E, 12.08.2017 granite boulder and old iron constructions in young pine forest

a3 SW shore, between capes Uglovoj (Ristiniemi) and Zubets (Kartinaisenniemi), 5959’49.3”N, 2747’42.8”E, open 16.08.2017 sandy seashore with Lathyrus maritimus L. and Rosa rugosa

a4 Central part, NE shore of Bol'shoe Lake (Suurjärvi), 59 59’49.7”N, 2749'53.0”'E, pine forest with granite boulders

13.08.2017

a5 S part, SE shore of Bol'shoe Lake (Suurjärvi), 59 59'19.7”N, 27 50'25.6”'E, sandy road in pine forest

13.08.2017

a6 Promezhutochny Peninsula (Suisaari), NNW part, central part of Vjunok Island (Mousoori), 6000’58.9”N, 14.08.2017 $27^{\circ} 52^{\prime} 45.7^{\prime \prime}$, open seashore

a7 Promezhutochny Peninsula (Suisaari), NNW part, S of Vjunok Island (Mousoori), 6000’42.7”N, 2752’52.2”E, 14.08.2017 seashore Phragmites australis (Cav.) Trin. ex Steud. community with Arthemisia vulgaris L. and Leymus arenarius (L.) Hochst., with single rowan and black alder trees

a8 Promezhutochny Peninsula (Suisaari), NW shore SW of Vjunok Island (Mousoori), 6000’34.7”N, 2752’58.7”E, 14.08.2017 seashore meadow

a) NW part of Hiekkataipale Isthmus, shore of Okol'naya Bay (Pohjois-Kappalahti), 6000'20.5”N, 2751'58.8”'E, open 14.08 .2017 sandy seashore with granite boulders

a10 N shore of Podkhodnoj Island (Kukkoori), 6002’04.1”N, $27^{\circ} 49^{\prime} 03.2^{\prime \prime} \mathrm{E}$, concrete constructions on seashore

a11 N of S part of Podkhodnoj Island (Kukkoori), 60 $01^{\prime} 56.7^{\prime \prime} \mathrm{N}, 27^{\circ} 49^{\prime} 11.4^{\prime \prime}$, seashore juniper community with granite 15.08 .2017 boulders

a12 N end, central part of Kirkorvi Spit (Kierkoori), 6001'54.1”N, 2749’40.5”E, open pine woodland with Avenella 15.08 .2017 flexuosa, lichens, mosses, and granite boulders

a13 SW part, S of Zarnitsa Bay (Lansteininlahti, Suurenhiedanlahti), 6000'05.1”N, 2748'32.0”'E, community of Calluna 16.08 .2017 vulgaris, lichens, and mosses on sandy road in pine forest

a14 SW shore, Cape Zubets (Kartinaisenniemi), 6000’09.2”N, 2748'13.4”E, open sandy seashore with Rosa rugosa and 16.08 .2017 Lathyrus maritima

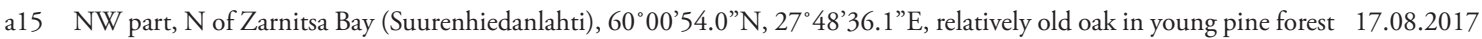
with birch and Calamagrostis sp.

a16 N part, S of Bay Malmihiianlahti, 6001'09.8”N, 2749'34.7”'E, old cemetery overgrown by young pine forest

a17 N part, W shore of Okol'naya Bay (Pohjois-Kappalahti), 6001’01.4”N, 2751’08.3”E, modern pier

a18 Central part, SW of Hiekkataipale Isthmus, 59 $59^{\prime} 54.3^{\prime \prime} \mathrm{N}, 27^{\circ} 50^{\prime} 47.1^{\prime \prime}$ E, young black alder stand surrounded by 20.08 .2017 spruce-pine forest 
a19 W part of Hiekkataipale Isthmus, $60^{\circ} 00^{\prime} 16.8^{\prime \prime} \mathrm{N}, 27^{\circ} 51^{\prime} 37.4$ 'E, wasteland on place of old aerodrome

a20 Central part, SW shore of Okol'naya Bay (Pohjois-Kappalahti), 6000'27.3”N, 2751'30.6”E, spruce-aspen forest with 22.08 .2017 Vaccinium myrtillus, Oxalis acetosella, and mosses, with abundant fallen deadwood

a21 Central part, Vehnämaa, $60^{\circ} 00^{\prime} 38.9^{\prime} \mathrm{N}, 27^{\circ} 49^{\prime} 57.8^{\prime \prime} \mathrm{E}$, old concrete construction covered by mosses and lichens

Historical localities:

BL1 W part of Hiekkataipale Isthmus, [60 $\left.00^{\prime} \mathrm{N}, 27^{\circ} 51-52^{\prime} \mathrm{E}\right]$, margin of sandy dune ('Die Randdüne. Die Dünensysteme 1926 westlich von Hiekkataipale') (same with locality 32)

BL2 E of Hiekkataipale Isthmus, Promezhutochny Peninsula (Suisaari), [6000’ N, 27 $52-53^{\prime} \mathrm{E}$ ], sandy dune covered with 1926 forest ('Die bewaldeten Dünen. Das Dünengebiet auf der N. Küste') (same with locality 5) 